\title{
Photoluminescence and Electrochemiluminescence of Thermally Activated Delayed Fluorescence (TADF) Emitters Containing Diphenylphosphine Chalcogenide-Substituted Carbazole Donors
}

Shiv Kumar, ${ }^{\dagger a}$ Pauline Tourneur ${ }^{+b}$ Jonathan R. Adsetts, ${ }^{\dagger c}$ Michael Y. Wong, ${ }^{a}$ Pachaiyappan Rajamalli, ${ }^{a}$ Dongyang Chen, ${ }^{a}$ Roberto Lazzaroni, ${ }^{b, d}$ Pascal Viville, ${ }^{d}$ David B. Cordes,${ }^{a}$ Alexandra M. Z. Slawin, ${ }^{a}$ Yoann Olivier, ${ }^{* e}$ Jérôme Cornil, ${ }^{* b}$ Zhifeng Ding ${ }^{* c}$ and Eli Zysman-Colman*a

${ }^{a}$ Organic Semiconductor Centre, EaStCHEM School of Chemistry, University of St Andrews, St Andrews, Fife, UK, KY16 9ST; E-mail: eli.zysman-colman@st-andrews.ac.uk; URL: http://www.zysman-colman.com; ${ }^{b}$ Laboratory for Chemistry of Novel Materials, University of Mons, Mons, Belgium; E-mail: Jerome.Cornil@umons.ac.be; URL: http://morris.umons.ac.be/;

${ }^{c}$ Department of Chemistry, The University of Western Ontario, London, Ontario N6A 3K7, Canada; E-mail: zfding@,uwo.ca; URL: https://publish.uwo.ca/ zfding/

${ }^{d}$ Materia Nova, Materials R\&D center, Mons, Belgium; E-mail:

Pascal.Viville@materianova.be; URL: http://www.materianova.be/

${ }^{e}$ Unité de Chimie Physique Théorique et Structurale (UCPTS) \& Laboratoire de Physique du Solide (LPS), Namur Institute of Structured Matter (NISM), University of Namur, Namur, Belgium; E-mail: yoann.olivier@unamur.be;

$(\dagger)$ Authors equally contributed.

\begin{abstract}
Aiming to develop efficient blue-emitting thermally activated delayed fluorescence (TADF) compounds, we have designed and synthesized derivatives of the well-known sky-blue emitter 2CzPN that contain electron-accepting phosphine chalcogenide groups to stabilize the HOMO level relative to the pristine compound, thus increasing the HOMO-LUMO gap and blueshifting the emission wavelength. By cyclic voltammetry, photophysical data and quantumchemical calculations, it was found that polar solvents and matrices validated the proposed concept, but these trends were not recovered in non-polar media. The suitability of these 2CzPN derivatives in polar matrices for optoelectronic applications was explored with electrochemiluminescence (ECL) by measuring emission delays, radical stability, emission stabilities, emission efficiencies and emission spectra. Some of the $2 \mathbf{C z P N}$ derivatives showed an unprecedented delayed onset of the ECL, and delayed rising time to the ECL maximum, as well as long ECL emission decay. All of these mentioned delay times suggest that these luminophores primarily emit via organic long-persistent electrochemiluminescence (OLECL) mechanisms. The derivatization of the donor groups of the emitters affected both the radical stability and the predominant emission mechanism, providing important insight into their potential as emitters in solid-state electroluminescent devices.
\end{abstract}

\section{Introduction}

Developing strategies to harvest triplet states in organic light-emitting diodes (OLEDs) based on small molecules has been the focus of considerable effort because, according to spin statistics, they constitute $75 \%$ of the excitons generated in the device. ${ }^{1}$ While phosphorescent heavy-metal-based organometallic complexes have successfully achieved 100\% internal quantum efficiency (IQE) thanks to their large spin-orbit coupling (SOC) and hence rapid 
intersystem crossing (ISC) rates, the use of scare elements such as iridium and platinum is a detracting feature that will ultimately impact large-scale application of OLED technology. ${ }^{2}$ Moreover, the inherent instability of blue-emitting heavy metal phosphorescent OLEDs has meant this class of materials is a non-starter in commercial devices. ${ }^{3}$

Materials showing thermally activated delayed fluorescence (TADF), which typically take the form of donor-acceptor organic compounds, have attracted tremendous recent attention as they are capable of efficiently harvesting triplet excitons. ${ }^{4-8}$ For the TADF mechanism to be operational, there must exist a suitably small splitting energy $\left(\Delta E_{\mathrm{ST}}\right)$ between the lowest excited singlet $\left(\mathrm{S}_{1}\right)$ and triplet $\left(\mathrm{T}_{1}\right)$ states. When this criterion is met, efficient reverse intersystem crossing (RISC) processes become feasible such that non-emissive triplet excitons are thermally up-converted to emissive singlet excitons. ${ }^{5,9-12}$ In order to maintain a small $\Delta E_{\mathrm{ST}}$, the exchange integral between the HOMO and LUMO must be minimized, ${ }^{13}$ and is commonly achieved by the spatial separation of these orbitals. ${ }^{8,12,14}$ In general, there are three main molecular designs that respond to this criterion: (1) compounds with twisted conformations between donor and acceptor moieties that emit via an intramolecular charge-transfer state (ICT) $;^{10}$ (2) compounds that promote through-space charge-transfer interactions between adjacent donor and acceptor units (TSCT) $;{ }^{15}$ and (3) compounds that are both p- and n-doped nanographenes that emit via multi-resonance TADF (MR-TADF). ${ }^{16}$ Each TADF emitter design principle has its own advantages and disadvantages in terms of emission color, color purity, photoluminescence quantum yield $\left(\Phi_{\mathrm{PL}}\right)$, and RISC efficiency, the latter of which is correlated strongly with device stability. For example, ICT- and TSCT-based TADF emitters have been reported to be both highly emissive; their color is easily tunable but their broad emission profiles lead to poor color purity. ${ }^{15,17-18}$ In contrast, MR-TADF emitters exhibit excellent color purity and $\Phi_{\mathrm{PL}}$ but generally possess much lower RISC rates. ${ }^{19}$ Regardless of emitter design, the stability of TADF OLEDs remains sub-optimal. ${ }^{6}$

In the pantheon of ICT emitter design, the majority of the research focus has been devoted to new acceptors while the selection of donor moieties has remained rather limited; with carbazole, ${ }^{10,20-21}$ triphenylamine, ${ }^{22-24}$ acridan, ${ }^{25-27}$ phenoxazine, ${ }^{28-31}$ phenothiazine ${ }^{32-33}$ and dihydrophenazine ${ }^{34-35}$ accounting for more that ca. $98 \%$ of all reported donors. This library of donors has been enriched through their chemical substitution that serves to tune the photophysical properties of the emitter. The majority of examples revolve around substituted carbazole derivatives and can be broadly categorized into two families: (i) substituents that enhance the electron-donating strength of carbazole and promote a red-shift in the emission, and; (ii) substituents that reduce the electron-donating strength of the carbazole and promote a blue-shift in the emission. ${ }^{36}$ Electron-rich carbazole derivatives used within TADF emitter design include 3,6-dimethylcarbazole,${ }^{37}$ 3,6-dimethoxycarbazole $,{ }^{38} 3,6$-di-t-butylcarbazole,${ }^{37-}$ ${ }^{38}$ fluorene-fused carbazole, ${ }^{39}$ benzofuran-fused carbazole, ${ }^{40}$ donor-dendronized carbazole ${ }^{41-42}$ benzothiophene-fused carbazole, ${ }^{43}$ and paracyclophane-fused carbazole. ${ }^{44}$ In contrast, 3,6diphenylcarbazole, ${ }^{10} \quad$ 9-phenylcarbazole,${ }^{45} \quad \alpha$ - $/ \beta$ - $/ \delta$-carboline, ${ }^{46-50} \quad$ indolocarbazole,${ }^{51}$ diphenylphosphine oxide-substituted carbazole derivatives, ${ }^{52}$ triaryl and diarylboronatesubstituted carbazole derivatives ${ }^{53}$ and cyanocarbazoles ${ }^{54-55}$ represent examples of electronpoor carbazole derivatives reported in the TADF literature. In the context of blue TADF emitters employing functionalized carbazole donors, Adachi et al. have reported several examples of blue TADF emitters. In 2012, Adachi et al. reported the first example of a deep blue emitter, DTC-DPS $\left(\lambda_{\mathrm{PL}}=423 \mathrm{~nm}, \Phi_{\mathrm{PL}}=80 \%, \Delta E_{\mathrm{ST}}=0.32 \mathrm{eV}\right.$ in $10 \mathrm{wt} \%$ in DPEPO), based on 3,6-di-(tert-butyl)carbazole as the donor and diphenylsulfone as the acceptor. The OLED employing this emitter showed a maximum external quantum efficiency $\left(\mathrm{EQE}_{\max }\right)$ of $9.9 \%$ and CIE coordinates of $(0.15,0.17) .{ }^{56}$ In a following report, an improved $\mathrm{EQE}_{\max }$ of $14.5 \%$ and CIE coordinates of $(0.16,0.16)$ were reported for the emitter, DMOC-DPS $\left(\lambda_{\mathrm{PL}}=\right.$ 
$455 \mathrm{~nm}, \Phi_{\mathrm{PL}}=80 \%, \Delta E_{\mathrm{ST}}=0.21 \mathrm{eV}$ in $10 \mathrm{wt} \%$ in DPEPO) in which 3,6-di-(tertbutyl)carbazole (DTC) was replaced by 3,6-dimethoxycarbozole (DMOC) as the donor. ${ }^{38}$ In another example, a sky-blue emitter BDPCC-TRZ $\left(\lambda_{\mathrm{PL}}=480 \mathrm{~nm}, \Phi_{\mathrm{PL}}=100 \%, \Delta E_{\mathrm{ST}}=0.11\right.$ $\mathrm{eV}$ in $6 \mathrm{wt} \%$ in DPEPO), composed of bis(3,6-diphenylcarbazolyl)carbazole (BDPCC) as the donor and triphenyl-1,3,5-triazine (TRZ) as the acceptor, was reported. The OLED with this emitter achieved an EQE $E_{\max }$ of $20.6 \%$ with $\lambda_{\mathrm{EL}}$ at $487 \mathrm{~nm}$ and CIE coordinates of $(0.19,0.25) .{ }^{57}$ Lee et al. reported 5,11-di(9H-carbazol-9-yl)indolo[3,2,1-jk]carbazole-2-carbonitrile $\left(\mathbf{C N I C C z}, \lambda_{\mathrm{PL}}=445 \mathrm{~nm}, \Phi_{\mathrm{PL}}=46 \%, \Delta E_{\mathrm{ST}}=0.27 \mathrm{eV}\right)$ as a deep blue emitter, with a CNmodified indolocarbazole as the acceptor core and carbazole as the donor. Here, deep-blue OLEDs $\left[\lambda_{\mathrm{EL}}=449 \mathrm{~nm}\right.$, CIE: $\left.(0.15,0.08)\right]$ were realized with an $\mathrm{EQE}_{\max }$ of $12.4 \% .{ }^{58}$

In the seminal paper published in 2012, Adachi et al. reported one of the earliest skyblue TADF emitters, 4,5-dicarbazolylphthalonitrile $\left(\mathbf{2 C z P N}, \lambda_{\mathrm{PL}}=473 \mathrm{~nm}, \Phi_{\mathrm{PL}}=47 \%\right.$ in toluene). ${ }^{10}$ An OLED device employing this emitter produced a sky-blue emission $\left(\lambda_{\mathrm{EL}}=475\right.$ $\mathrm{nm}$ in $5 \mathrm{wt} \%$ PPT doped emitting layer) with an EQE $\mathrm{max}_{\max }$ of $8.0 \%$. An improved OLED device performance $\left(\lambda_{\mathrm{EL}}=483 \mathrm{~nm}\right.$, CIE: $\left.(0.17,0.30), \mathrm{EQE}_{\max }=13.6 \%\right)$ was achieved when the same emitter was used in a $\mathrm{mCP}$ host $\left(\lambda_{\mathrm{PL}}=477 \mathrm{~nm}, \Phi_{\mathrm{PL}}=89 \%\right.$ in $6 \mathrm{wt} \% \mathrm{mCP}$ doped film, $\Delta E_{\mathrm{ST}}=$ $0.09 \mathrm{eV}) .{ }^{59}$ By optimizing the device architecture, Sun et al. were able to achieve an EQE $\mathrm{max}_{\max }$ of $21.8 \%$ [CIE: $(0.17,0.27), \lambda_{\mathrm{EL}}=480 \mathrm{~nm}$ ] while using the same emitter in the OLED, by adopting a mixed co-host system (mCP:PO15 = 1:1). However, such a high EQE was only achieved at a very low current density of ca. $0.01 \mathrm{~mA} \mathrm{~cm}^{-2}$, and the device suffered from severe efficiency roll-off at high current density ${ }^{58}$ In pursuit of a deeper blue color using the same general structural motif as $\mathbf{2 C z P N}$, Gyeong et al. reported $\boldsymbol{\alpha}-\mathbf{C b P N}\left(\lambda_{\mathrm{PL}}=445 \mathrm{~nm}, \Phi_{\mathrm{PL}}=37 \%\right.$, $\Delta E_{\mathrm{ST}}=0.28 \mathrm{eV}$ in $20 \mathrm{wt} \% \mathrm{mCP}$ doped film) and $\boldsymbol{\delta}-\mathbf{C b P N}\left(\lambda_{\mathrm{PL}}=480 \mathrm{~nm}, \Phi_{\mathrm{PL}}=93 \%, \Delta E_{\mathrm{ST}}\right.$ $=0.13 \mathrm{eV}$ in $20 \mathrm{wt} \% \mathrm{mCP}$ doped film), employing $\alpha / \delta$-carboline donors in lieu of carbazole. ${ }^{60}$ A maximum EQE of $22.5 \%$ for a sky-blue OLED with $\lambda_{\mathrm{EL}}=486 \mathrm{~nm}$ and CIE: $(0.19,0.34)$ was achieved using $20 \mathrm{wt} \% \boldsymbol{\delta}$-CbPN in $\mathrm{mCP}$ as the light-emitting layer. The same group also reported a triazine-based emitter 5,5-(2-(9H-carbazol-9-yl)-5-(4,6-diphenyl-1,3,5-triazin-2yl)-1,3-phenylene)bis(5H-pyrido[3,2-b]indole) (CzDCbTrz) containing a $\delta$-carboline donor, and produced an OLED with an $\mathrm{EQE}_{\max }$ of $23.4 \%$ and $\lambda_{\mathrm{EL}}=471 \mathrm{~nm}$ with CIE: $0.16,0.19$ using $6 \mathrm{wt} \%$ emitter in 2,8-bis(diphenylphosphineoxide)-dibenzofuran (DBFPO) host.

Electrochemiluminescence (ECL) or electrogenerated chemiluminescence is emitted when electron transfer between electrochemically-generated radicals results in an exciton formation in the proximity of a working electrode, which relaxes radiatively. ${ }^{61-64}$ In this sense, the mechanism of light generation via ECL mirrors that in an OLED. This technique finds additional applications in discovering luminophore film enhancement phenomena, ${ }^{65-67}$ and in commercial applications for antigen sensing. ${ }^{68}$ Since the early studies on ECL in the $1960 \mathrm{~s},{ }^{69-}$ 71 various types of emissive compounds have been studied. ${ }^{72}$ However, the design of commercial ECL luminophores has been mainly limited to noble-metal complexes such as those of $\mathrm{Ru}$, due to their efficient and electrochemically-stable conversion of excitons to light, regardless of the total spin. Analogously to their popular use as emitters in OLEDs, TADF molecules are likewise expected to give high ECL efficiency. Despite the thousands of reports of TADF emitters used in OLEDs, there are only a small number of examples of TADF compounds employed in ECL studies. Imato et al. studied the annihilation ECL properties of carbazole-decorated phthalonitrile based TADF emitters in both DCM and MeCN. ${ }^{73}$ Of the compounds investigated, the ECL emission maxima, $\lambda_{\mathrm{ECL}}$, for $2 \mathbf{C z P N}$ were modestly redshifted $\left(\lambda_{\mathrm{ECL}}=550\right.$ and $585 \mathrm{~nm}$ in $\mathrm{DCM}$ and $\mathrm{MeCN}$, respectively) compared to the photoluminescence maxima, $\lambda_{\mathrm{PL}},\left(\lambda_{\mathrm{PL}}=533\right.$ and $560 \mathrm{~nm}$ in DCM and MeCN, respectively) regardless of solvent. Further, the low $\Phi_{\mathrm{PL}}\left(\Phi_{\mathrm{PL}}=34\right.$ and $10 \%$ in $\mathrm{DCM}$ and $\mathrm{MeCN}$, respectively) translated into relatively low ECL efficiencies, $\Phi_{\mathrm{ECL}}$, of 90 and $22 \%$, respectively, 
in $\mathrm{DCM}$ and $\mathrm{MeCN}$, in comparison to $\left[\mathrm{Ru}(\mathrm{bpy})_{3}\right]^{2+}$, which showed $\Phi_{\mathrm{ECL}}=100 \%$ under the same conditions. No discussion concerning the kinetics of exciton decay was provided. Most recently, Niu et al. reported the first example of a solid-state ECL study of a TADF polymer containing a backbone acridan-based donor and pendant triazine-containing acceptor, PCzAPT10. ${ }^{74}$ The polymer showed significantly enhanced $\Phi_{\mathrm{ECL}}$ of $194 \%$ relative to $\left[\mathrm{Ru}(\mathrm{bpy})_{3}\right]^{2+}$ when using tri-n-propylamine (TPA) as a co-reactant, compared to the $\Phi_{\mathrm{PL}}$ of $21 \%$. The $\lambda_{\text {ECL }}$ was also significantly red-shifted, at $587 \mathrm{~nm}$, compared to the $\lambda_{\mathrm{PL}}(542 \mathrm{~nm})$ for a neat film of PCzAPT10 immersed in 0.1 M TBAP:MeCN electrolyte solution. Furthermore, Niu et al. made water-soluble 4CzIPN nanoparticles which achieved a relative $\Phi_{\mathrm{ECL}}=0.7 \%$, marking the first water-soluble TADF nanoparticle tested. ${ }^{75}$

Using the same $\mathbf{2 C z P N}$ scaffold as a starting point, we hypothesized that adding strong electron-withdrawing group such as phosphine-chalcogenides onto the carbazole donor moieties would promote the desired blue-shift in the emission. In this regard, we designed and synthesized three 2CzPN derivatives and thoroughly investigated their physical, photophysical, electrochemical properties, further supporting these optoelectronic studies with density functional theory (DFT) calculations (Scheme 1). ECL was then used to gauge the suitability of these derivatives for optoelectronic applications in the desirable polar media. Assessing the potential of the three $2 \mathbf{C z P N}$ derivatives as OLED materials was possible without the time-intensive and costly task of constructing full OLEDs, since ECL provides a tool to simulate charge imbalances, judge relative stabilities of electrically generated radical cations and anions (holes and electrons, respectively), evaluate relative emission efficiencies and understand emission mechanisms of luminophores. ${ }^{73}$, 76-77 Although our reference compound, 2CzPN is a well-studied TADF emitter in the literature, we have redetermined its optoelectronic properties under the same conditions as the three new emitters, as well as calculating its properties, to allow the most accurate comparison.

\section{Results and Discussion}

\subsection{Synthesis and Characterization}

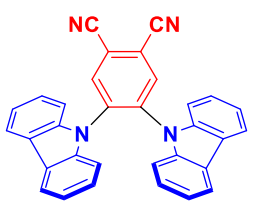

2CzPN

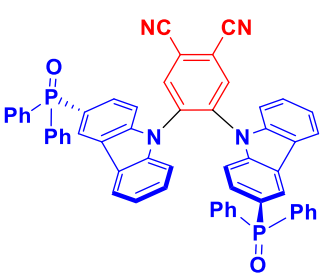

PPOCzPN

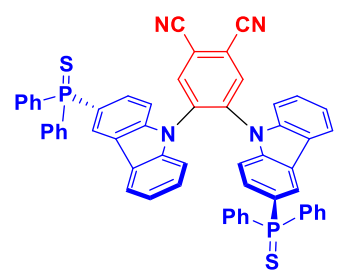

PPSCzPN

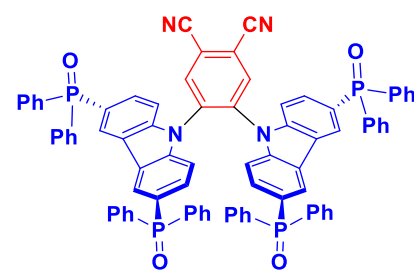

DiPPOCzPN

Scheme 1. Chemical structure of $\mathbf{2 C z P N}$ and the three phosphine chalcogenide derivatives.

The synthesis of the phosphine chalcogenide-modified 2CzPN emitters is shown in Scheme 2. Nitrogen-protected precursors 9-(tert-butyldimethylsilyl)-3-bromo-9H-carbazole, TBDMS-BrCz and 9-(tert-butyldimethylsilyl)-3,6-dibromo-9H-carbazole, TBDMS-DBrCz were prepared from their respective bromo- or dibromo- $9 H$-carbazole derivatives. ${ }^{78}$ The key intermediates, the phosphine chalcogenide-substituted carbazoles (PPOCz, PPSCz, DiPPOCz), were prepared by lithiation of TBDMS-BrCz and TBDMS-DBrCz followed by diphenylphosphination

(TBDMS-PPCz and TBDMS-DiPPCz), and finally 
oxidation/sulfuration. Removal of the TBDMS group afforded the desired phosphine chalcogenide-substituted carbazole derivatives in moderate-to-excellent yield (40-95\%). The respective emitters PPOCzPN, PPSCzPN and DiPPOCzPN were likewise obtained in moderate-to-excellent yield (52-96\%) through a nucleophilic aromatic substitution reaction of 4,5-difluorophthalonitrile with PPOCz, PPSCz or DiPPOCz. All the emitters were characterized by ${ }^{1} \mathrm{H},{ }^{13} \mathrm{C}$, and ${ }^{31} \mathrm{P}$ NMR spectroscopy and high-resolution mass spectrometry (HRMS), and their purity was demonstrated by HPLC and elemental analysis. The emitters PPOCzPN and PPSCzPN were each found to exist as a mixture of rotamers at room temperature in a ratio of 58:42 and 70:30, respectively, which was observed by both HPLC and ${ }^{31} \mathrm{P}$ NMR spectroscopy (Figure S1). This was further substantiated by obtaining single crystals of both rotamers for PPSCzPN.

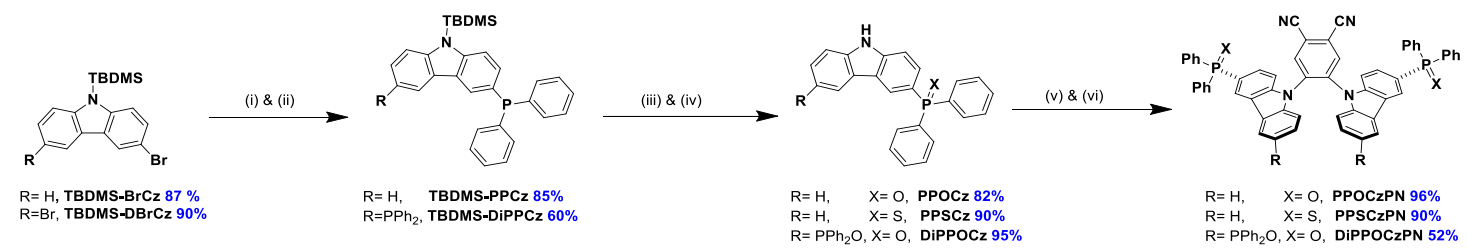

Scheme 2. Synthetic routes for the synthesis of PPXCzPN and DiPPOCzPN. Reaction conditions: (i) $n$ - $\mathrm{BuLi}(1.6 \mathrm{M}), \mathrm{THF},-78^{\circ} \mathrm{C}, 30 \mathrm{~min}$, (ii) $\mathrm{PPh}_{2} \mathrm{Cl},-78{ }^{\circ} \mathrm{C}$ - rt, $30 \mathrm{~min}$, (iii) $\mathrm{H}_{2} \mathrm{O}_{2}$ or $\mathrm{S}_{8}, \mathrm{THF}, \mathrm{rt}, 18 \mathrm{~h}$, (iv) TBAF $(1.0 \mathrm{M})$, THF, rt, 1h, (v) NaH, THF, $30 \mathrm{~min}$, (vi) 4,5difluorophthalonitrile, rt, $6 \mathrm{~h}$.

\section{Single Crystal X-ray Diffraction}



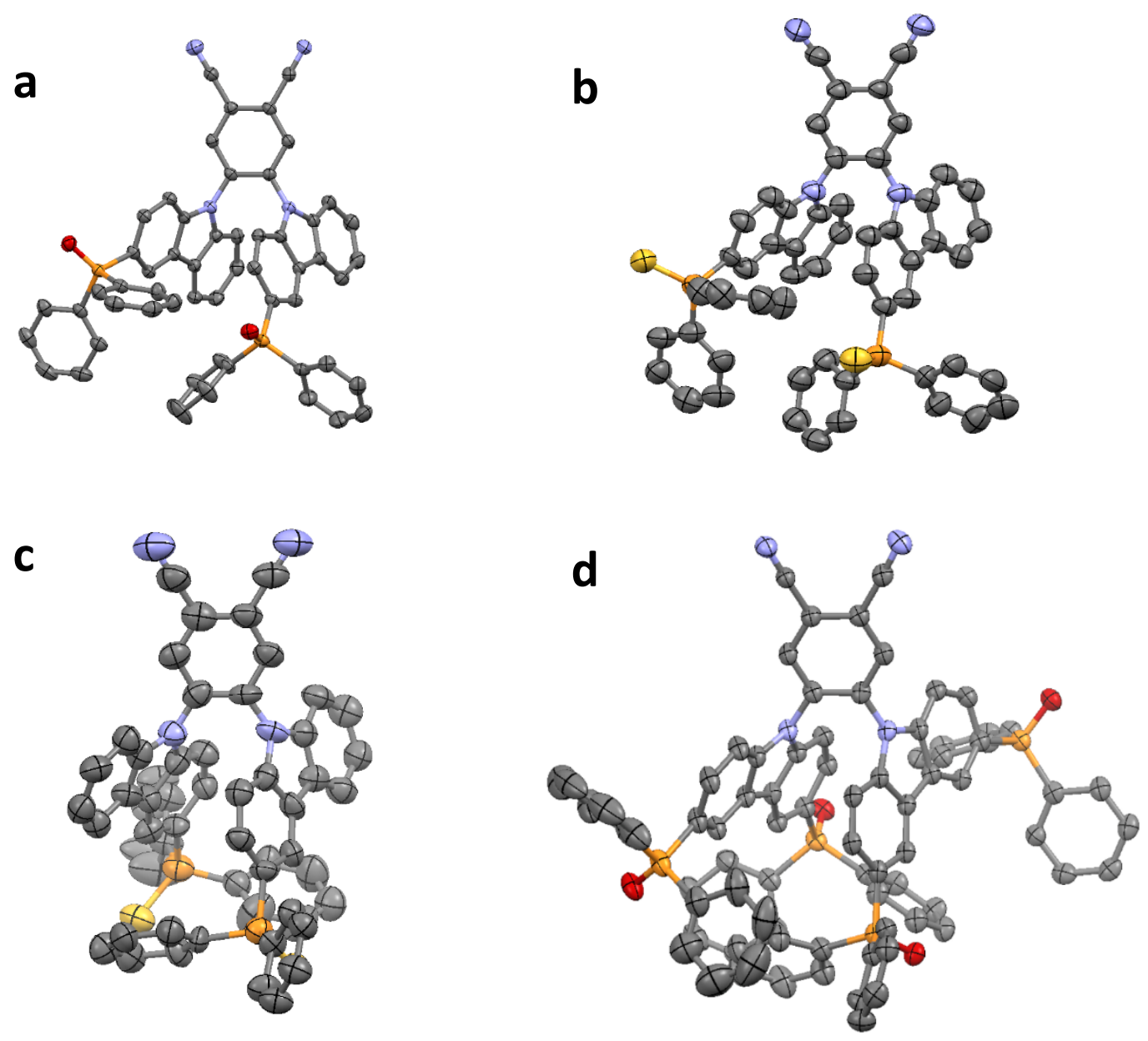

Figure 1. Thermal ellipsoid plots of the crystal structures of (a) PPOCzPN, (b) PPSCzPN-1, (c) PPSCzPN-2 and (d) DiPPOCzPN. Ellipsoids are drawn at the $50 \%$ probability level and hydrogen atoms and solvent molecules are omitted for clarity.

The molecular structures of PPOCzPN, PPSCzPN, and DiPPOCzPN were determined by single crystal X-ray diffraction analysis (Figure 1). Single crystals were grown from a binary solvent system by the vapour diffusion method: chloroform/acetonitrile and DCM/hexane for PPOCzPN; DCM/hexane and chloroform/methanol for PPSCzPN; and DCM/hexane for DiPPOCzPN. On initial analysis of the structure of PPOCzPN (obtained from chloroform/acetonitrile), partial-occupancy chlorine atoms were identified on both the carbazoles, forming PPOCICzPN. This likely arose from chlorination during crystallization from the chloroform solvent. A later crystallisation from DCM/hexane gave rise to a structure of PPOCzPN not showing any chlorination. Crystallisation of PPSCzPN from the two different solvent combinations gave rise to crystals showing substantially different unit cells. Analysis of these revealed the presence of a different rotamer in each structure, with form 2 also showing included solvent. Only a single rotamer could be crystallised for PPOCzPN. In all the emitters, the interplanar angles between the donor carbazole derivatives and phthalonitrile moiety ranged between $50.7^{\circ}$ and $59.7^{\circ}$ and were mostly close to identical within an individual molecule [PPOCzPN 58.12(6) ${ }^{\circ}$ and 59.36(6) ${ }^{\circ}$, PPSCzPN-1 50.7(3) ${ }^{\circ}$ and $50.9(3)^{\circ}$, PPSCzPN-2 52.8(8) ${ }^{\circ}$ and 53.9(8) ${ }^{\circ}$, and 51.5(7) ${ }^{\circ}$ and $59.7(7)^{\circ}$, and DiPPOCzPN $56.82(8)^{\circ}$ and 57.69(8)]. By contrast, a larger interplanar angle range is computed in the optimized ground-state geometries of the isolated emitters with all compounds showing one torsion angle around $45^{\circ}$ and the other around $70^{\circ}$ (see Section 2.3). 
The four structures show a number of intermolecular interactions. In both PPOCzPN and DiPPOCzPN a three-dimensional network of weak $\mathrm{C}-\mathrm{H} \cdots \mathrm{O}$ and $\mathrm{C}-\mathrm{H} \cdots \mathrm{N}$ hydrogen bonded molecules is formed [PPOCzPN H*A distances 2.39-2.60 $\AA, \mathrm{C} \cdots \mathrm{A}$ separations

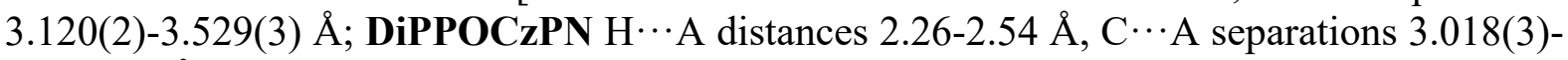
3.383(3) $\AA$ ] (where $\mathrm{A}$ is the acceptor atom). The networks are reinforced by $\mathrm{C}-\mathrm{H} \cdots \pi$

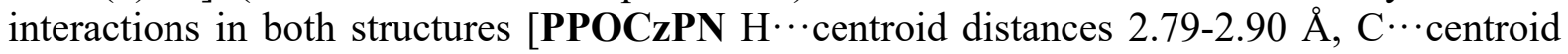

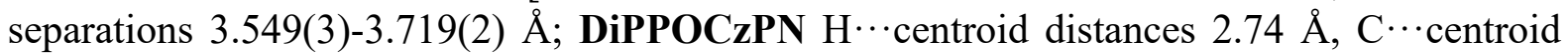
separations 3.546(3) $\AA$ ], $\pi-\pi$ interactions in PPOCzPN [centroid $\cdots$ centroid distances 3.7287(9) $\AA$ ], and by conventional hydrogen bonds to the water solvent molecule in DiPPOCzPN $[\mathrm{H} \cdots \mathrm{O}$ distances 1.83(3) and 1.87(4) $\AA, \mathrm{O} \cdots \mathrm{O}$ separations 2.811(3) and 2.858(4) $\AA]$.

Both PPSCzPN structures show a different pattern of interactions where weak interactions of specific types give rise to multiple one-dimensional chain structures, these being linked into two-dimensional sheets when they are combined. In PPSCzPN-1, C-H $\cdots \pi$ interactions $[\mathrm{H} \cdots$ centroid distances 2.70 and $2.89 \AA, \mathrm{C} \cdots$ centroid separations 3.623(12) and $3.799(15) \AA$ ] form chains along the ac-diagonal axis, and weak $\mathrm{C}-\mathrm{H} \cdots \mathrm{N}$ hydrogen bonds $[\mathrm{H} \cdots \mathrm{N}$ distances $2.45 \AA, \mathrm{C} \cdots \mathrm{N}$ separations $3.322(16) \AA]$ form chains along the $b$-axis. The combination of these leads to sheets in the (1 $10-1)$ plane. In PPSCzPN-2, $\pi \cdots \pi$ interactions [centroid $\cdots$ centroid distances 3.668(10)-3.803(10) $\AA$ ] form chains along the $b$-axis, and C$\mathrm{H} \cdots \pi$ interactions $[\mathrm{H} \cdots$ centroid distances $2.78 \AA, \mathrm{C} \cdots$ centroid separations $3.63(3) \AA]$ form chains along the $b c$-diagonal axis. The combination of these leads to sheets in the $\left(\begin{array}{lll}1 & 0 & 0\end{array}\right)$ plane, which are further reinforced by weak $\mathrm{C}-\mathrm{H} \cdots \mathrm{S}$ hydrogen bonds $[\mathrm{H} \cdots \mathrm{S}$ distances $2.71 \AA$, $\mathrm{C} \cdots \mathrm{S}$ separations 3.56(2) $\AA$ ]. The chloroform solvent molecules in PPSCzPN-2 are held within the lattice by weak hydrogen bonds $[\mathrm{H} \cdots \mathrm{N}$ distances $2.54 \AA$, C $\cdots \mathrm{N}$ separations 3.41(4) $\AA$; $\mathrm{H} \cdots \mathrm{S}$ distances $2.62 \AA, \mathrm{C} \cdots \mathrm{S}$ separations 3.59(2) $\AA]$.

None of these structures show a matching pattern of interactions to that shown in the structure of $\mathbf{2 C z P N}$, where multiple sets of $\mathrm{C}-\mathrm{H} \cdots \pi$ interactions form sheets in the $\left(\begin{array}{lll}0 & 0 & 1\end{array}\right)$ plane, which are reinforced by weak $\mathrm{C}-\mathrm{H} \cdots \mathrm{N}$ hydrogen bonds. ${ }^{79}$ This is closest to what is seen in PPSCzPN-1, in that sheets are formed by combinations of $\mathrm{C}-\mathrm{H} \cdots \pi$ and $\mathrm{C}-\mathrm{H} \cdots \mathrm{N}$ interactions; however, in $\mathbf{2 C z P N}$, the $\mathrm{C}-\mathrm{H} \cdots \pi$ interactions form sheets directly, whereas in PPSCzPN-1 both the C-H $\cdots \pi$ interactions and the weak hydrogen bonds are required to form the sheets. 


\subsection{Electrochemical and Photophysical Properties}
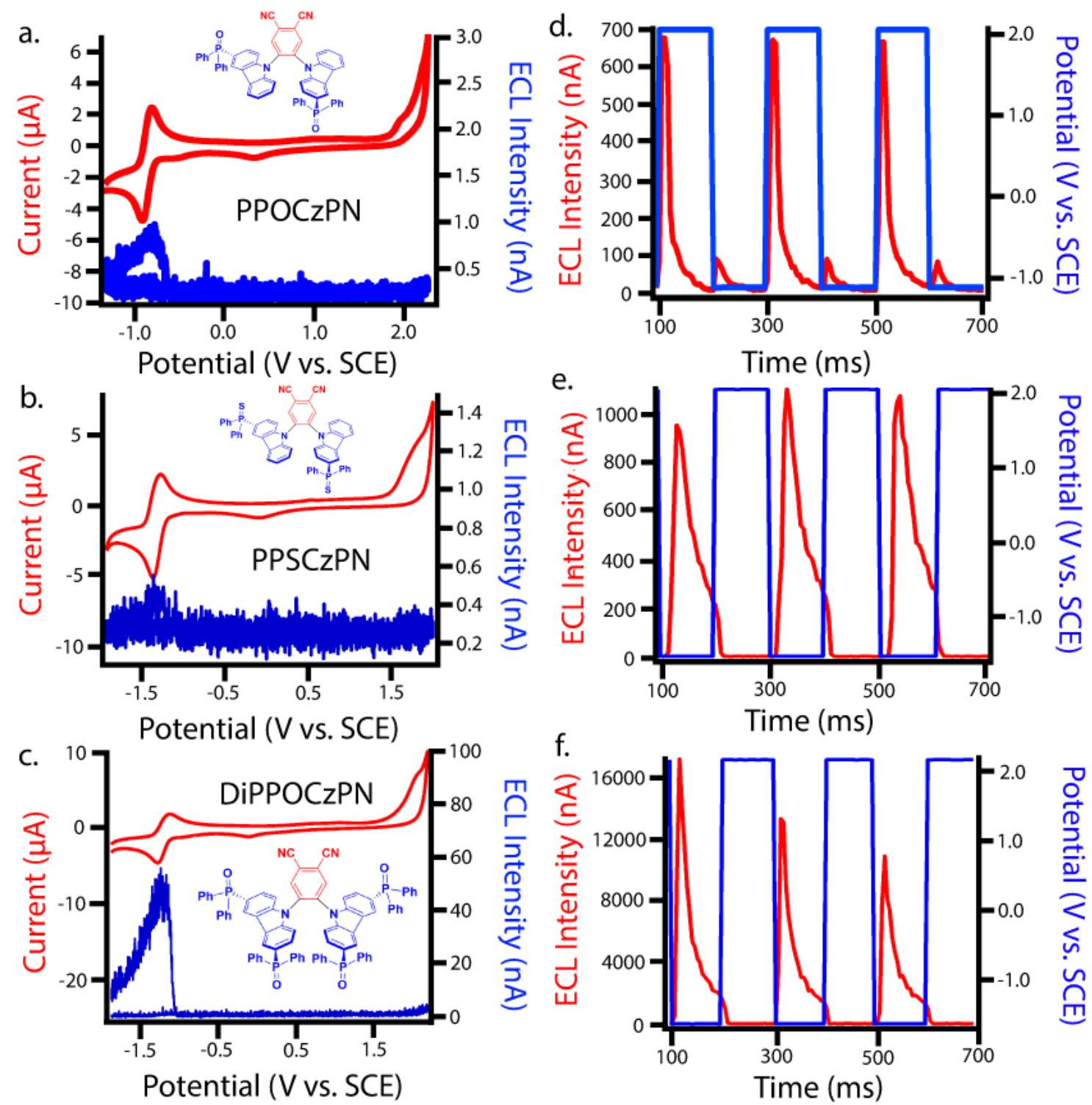

Figure 2. (a-c) CVs (red) along with ECL-voltage curves (blue) and (d-f) ECL-time curves (red) along with voltage-time curves (blue) during potential pulsing at a pulsing frequency of $10 \mathrm{~Hz}$ for PPOCzPN, PPSCzPN and DiPPOCzPN, respectively. Scans were recorded in DCM. CVs were all performed at a scan rate of $0.1 \mathrm{~V} / \mathrm{s}$. Pulsing experiments were carried out between 0.1 $\mathrm{V}$ above the first oxidation and below the first reduction potentials of the compounds.

The three emitters PPOCzPN, PPSCzPN and DiPPOCzPN show irreversible oxidation waves and reversible reduction waves in their cyclic voltammograms $(\mathrm{CV})$, recorded in DCM with $0.1 \mathrm{M}$ tetrabutylammonium perchlorate (TBAP) (red curves in Figure 2a-c). The values of the HOMO and LUMO energy levels were estimated from the peaks observed in the $\mathrm{CV}$ and verified from the differential pulse voltammograms (Figure S36). The HOMO/LUMO levels of the reference compound 2CzPN, as well as PPOCzPN, PPSCzPN and DiPPOCzPN are determined to be $-6.28 /-3.50,-6.76 /-3.93,-6.56 /-3.48$ and $-6.86 /-3.56 \mathrm{eV}$, respectively. As expected, it is harder to reduce DiPPOCzPN than PPOCzPN due to the presence of the second electron-withdrawing diphenylphosphine oxide moiety on each carbazole. PPOCzPN is the 
easiest to oxidize and has the least stable HOMO. Similar electrochemical trends were seen in MeCN (Figure S37).
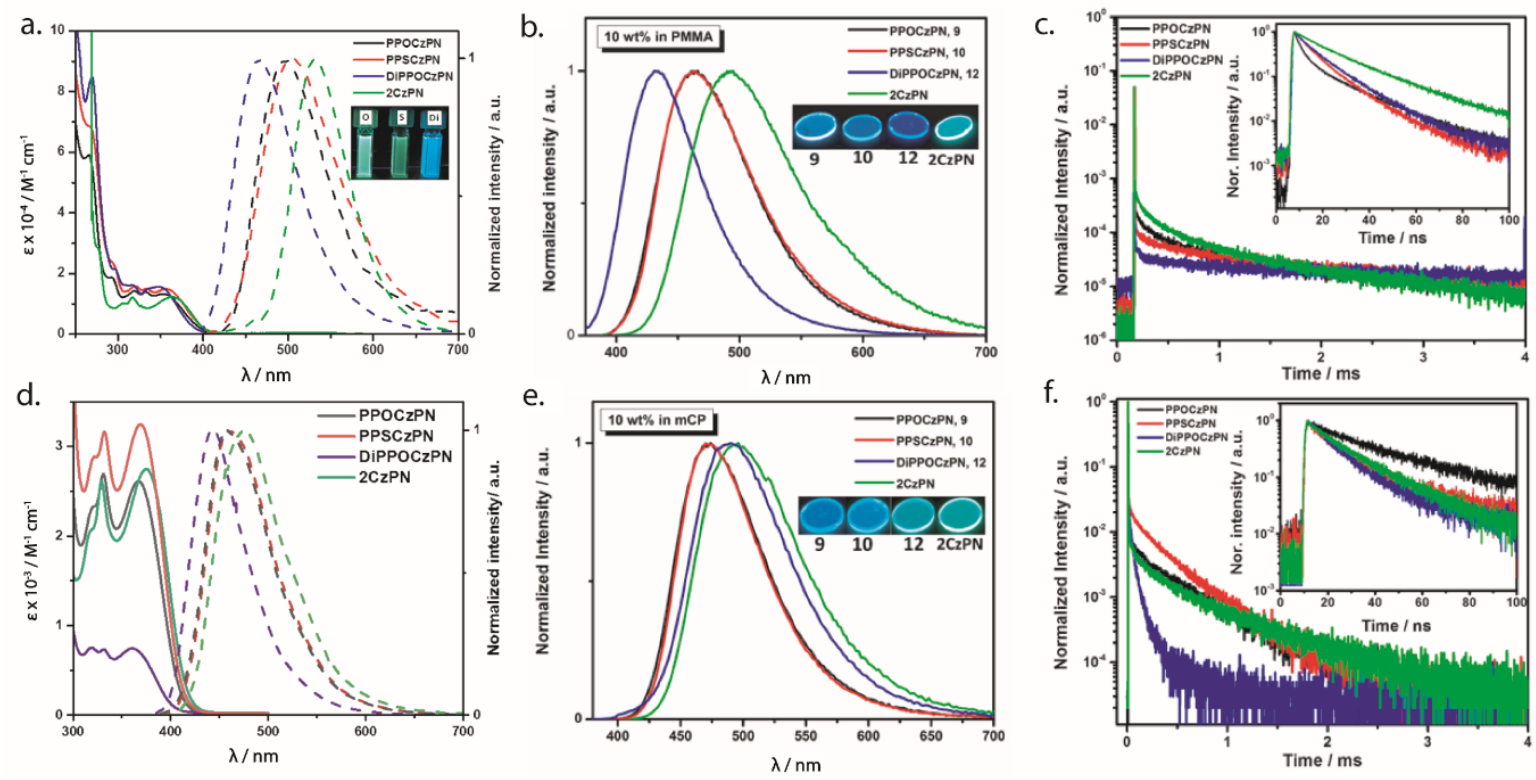

Figure 3. (a) Absorption (solid line) and emission (dashed line) spectra of PPOCzPN, PPSCzPN, DiPPOCzPN and 2CzPN recorded at room temperature in MeCN and DCM solution $\left(\lambda_{\mathrm{exc}}=358 \mathrm{~nm}\right)$, respectively. (b) Steady-state photoluminescence spectra of $10 \mathrm{wt} \%$ PMMA films of PPOCzPN, PPSCzPN, DiPPOCzPN, 2CzPN $\left(\lambda_{\text {exc }}=358 \mathrm{~nm}\right)$. (c) Timeresolved photoluminescence decay profiles of $10 \mathrm{wt} \%$ PMMA films of PPOCzPN, PPSCzPN, DiPPOCzPN, 2CzPN $\left(\lambda_{\mathrm{exc}}=378 \mathrm{~nm}\right)$. (d) Absorption and emission spectra of PPOCzPN, PPSCzPN, DiPPOCzPN and $2 \mathrm{CzPN}$ recorded at room temperature in toluene solution $\left(\lambda_{\text {exc }}=\right.$ $370 \mathrm{~nm}$ ). (e) Steady-state photoluminescence spectra of $10 \mathrm{wt} \% \mathrm{mCP}$ films of PPOCzPN, PPSCzPN, DiPPOCzPN, 2CzPN $\left(\lambda_{\text {exc }}=358 \mathrm{~nm}\right)$. (f) Time-resolved photoluminescence decay profiles of $10 \mathrm{wt} \% \mathrm{mCP}$ films of PPOCzPN, PPSCzPN, DiPPOCzPN, 2CzPN $\left(\lambda_{\mathrm{exc}}=\right.$ $378 \mathrm{~nm}$ ). 
Table 1. Summary of photophysical and electrochemical properties of PPOCzPN, PPSCzPN, DiPPOCzPN and 2CzPN in polar media and in a PMMA matrix.

\begin{tabular}{|c|c|c|c|c|c|c|c|c|}
\hline \multirow[b]{2}{*}{ Emitter } & \multirow{2}{*}{$\begin{array}{l}\mathrm{MeCN} \\
{ }_{\text {abs }}{ }^{a} / \mathrm{nm}, \\
\left(\varepsilon \times 10^{-4} / \mathrm{M}^{-1} \mathrm{~cm}^{-}\right. \\
1)\end{array}$} & \multicolumn{2}{|l|}{ DCM } & \multicolumn{2}{|c|}{$\begin{array}{l}10 \mathrm{wt} \% \text { doped PMMA } \\
\text { film }\end{array}$} & \multicolumn{3}{|l|}{ DCM } \\
\hline & & $\lambda_{\mathrm{PL}}^{b} / \mathrm{nm}$ & $\begin{array}{l}\Phi_{\mathrm{PL}}^{c} \\
/ \%\end{array}$ & $\lambda_{\mathrm{PL}}{ }^{d} / \mathrm{nm}$ & $\begin{array}{l}\Phi_{\mathrm{PL}}^{e} \\
/ \%\end{array}$ & $\begin{array}{l}\mathbf{E}_{\mathbf{o x}} / \mathrm{V} \text { (HOMO }^{f} / \\
\mathrm{eV})\end{array}$ & $\begin{array}{l}\mathbf{E}_{\text {red }} / \mathbf{V}\left(\mathbf{L U M O}^{f} /\right. \\
\mathrm{eV})\end{array}$ & $\begin{array}{l}\Delta E^{g} \\
/ \mathbf{e V}\end{array}$ \\
\hline PPOCzPN & $356(1.24)$ & 498 & 51 & 465 & 56 & $1.96(-6.76)$ & $-0.87(-3.93)$ & 2.83 \\
\hline PPSCzPN & $358(1.49)$ & 501 & 47 & 465 & 59 & $1.76(-6.56)$ & $-1.32(-3.48)$ & 3.08 \\
\hline DiPPOCzPN & $351(1.51)$ & 465 & 61 & 428 & 28 & $2.06(-6.86)$ & $-1.24(-3.56)$ & 3.29 \\
\hline $2 \mathrm{CzPN}$ & $366(1.33)$ & 515 & 29 & 494 & 77 & $1.48(-6.28)$ & $-1.30(-3.50)$ & 2.78 \\
\hline $2 \mathrm{CzPN}^{h}$ & $364(1.14)$ & 532 & 29 & 492 & 76 & NA $(-5.89)^{i}$ & $\mathrm{NA}(-2.97)^{i}$ & $2.92^{i}$ \\
\hline
\end{tabular}

${ }^{a}$ ICT band measured in MeCN at $298 \mathrm{~K}^{b}{ }^{b}$ In degassed DCM $\left(\lambda_{\mathrm{exc}}=358 \mathrm{~nm}\right) .{ }^{c}$ Solution $\Phi_{\mathrm{PL}}$ values were determined by the relative method ${ }^{4}$ using $0.5 \mathrm{M}$ quinine sulfate in $\mathrm{H}_{2} \mathrm{SO}_{4}(\mathrm{aq})$ as the reference $\left(\Phi_{\mathrm{PL}}: 54.6 \%\right) .{ }^{80}{ }^{d} 10 \mathrm{wt} \% \mathrm{PMMA}$ doped thin films $\left(\lambda_{\text {exc }}=360 \mathrm{~nm}\right) .{ }^{e}$ Absolute $\Phi_{\mathrm{PL}}$ values in $10 \mathrm{wt} \%$ PMMA doped thin films under $\mathrm{N}_{2} \cdot{ }^{f}$ In DCM with $0.1 \mathrm{M}\left[n-\mathrm{Bu}_{4} \mathrm{~N}\right] \mathrm{ClO}_{4}$ as the supporting electrolyte and $\mathrm{Fc} / \mathrm{Fc}^{+}$as the internal reference (0.48 V vs SCE) unless otherwise noted. ${ }^{81}$ The HOMO and LUMO energies were calculated using the relation $E_{\mathrm{HOMO}} / E_{\mathrm{LUMO}}=-\left(E^{\mathrm{ox}} / E^{\mathrm{red}}+4.8\right)$ $\mathrm{eV}$, where the $E^{\mathrm{ox}}$ and $E^{\mathrm{red}}$ are anodic and cathodic formal potentials, respectively, obtained from $\mathrm{CV} \cdot{ }^{82}{ }^{g} \Delta E=\left|E_{\mathrm{HOMO}}-E_{\mathrm{LUMO}}\right| \cdot{ }^{h}$ Values from ref 77. ${ }^{i}$ Determined in MeCN. NA = not available. 
Table 2. Summary of photophysical and electrochemical properties of PPOCzPN, PPSCzPN, DiPPOCzPN and 2CzPN in apolar media and in the solid state in a mCP matrix and as neat films.

\begin{tabular}{|c|c|c|c|c|c|c|c|c|}
\hline & \multicolumn{3}{|l|}{ Toluene } & \multicolumn{3}{|c|}{$10 \mathrm{wt} \%$ doped mCP film } & \multicolumn{2}{|c|}{ Neat film } \\
\hline Emitter & $\lambda_{\text {abs }}{ }^{a} / \mathbf{n m}$ & $\lambda_{\mathrm{PL}}^{b} / \mathrm{nm}$ & $\Delta E_{\mathrm{ST}}{ }^{g} / \mathrm{eV}$ & $\lambda_{\mathrm{PL}}^{c} / \mathbf{n m}$ & $\begin{array}{l}\Phi_{\mathrm{PL}}^{d} \\
/ \%\end{array}$ & $\Delta E_{\mathrm{ST}}^{g} / \mathrm{eV}$ & $\lambda_{\mathrm{PL}}{ }^{e} / \mathrm{nm}$ & $\begin{array}{l}\Phi_{\mathrm{PL}}^{f} \\
/ \%\end{array}$ \\
\hline PPOCzPN & $321,331,367$ & 460 & 0.20 & 474 & 72 & 0.19 & 501 & 54 \\
\hline PPSCzPN & $321,332,369$ & 460 & 0.42 & 474 & 73 & 0.24 & 524 & 26 \\
\hline DiPPOCzPN & $319,332,361$ & 443 & 0.43 & 490 & 56 & 0.15 & 466 & 38 \\
\hline $2 \mathrm{CzPN}$ & $317,329,375$ & 476 & 0.36 & 496 & 77 & 0.23 & 516 & 33 \\
\hline 2CzPN & $317,329,375^{h}$ & $484^{h}$ & $0.31^{h}$ & $477^{i}$ & $89^{i}$ & $0.09^{i}$ & NA & NA \\
\hline
\end{tabular}

${ }^{a}$ ICT band measured in toluene at $298 \mathrm{~K} .{ }^{b}$ In toluene $\left(\lambda_{\mathrm{exc}}=370 \mathrm{~nm}\right) .{ }^{c}$ In $10 \mathrm{wt} \% \mathrm{mCP}$ doped thin films. ${ }^{d}$ Absolute quantum yield in $10 \mathrm{wt} \%$ mCP doped thin films. ${ }^{e}$ In neat thin films. ${ }^{f}$ Absolute quantum yield in neat thin films. ${ }^{g}$ Measured as the energy difference between the prompt fluorescence onset (at $77 \mathrm{~K}, \lambda_{\mathrm{exc}}=378 \mathrm{~nm}$, time delay $=1 \mathrm{~ns}$, integration time: $1-100 \mathrm{~ns}$ ) and the phosphorescence onset $\left(\right.$ at $77 \mathrm{~K}, \lambda_{\text {exc }}=378 \mathrm{~nm}$, time delay $=1 \mathrm{~ms}$, integration time $=1-10 \mathrm{~ms}$ ). ${ }^{h}$ Values from eff $^{79} .{ }^{i}$ Values from ref ${ }^{59}$, measured in $6 \mathrm{wt} \%$ doped mCP film, $\Delta E_{\mathrm{ST}}$ was estimated from the fluorescence $(300 \mathrm{~K})$ and phosphorescence $(5 \mathrm{~K})$ peaks. NA = not available 
The optical absorption study of PPOCzPN, PPSCzPN, DiPPOCzPN and the reference emitter 2CzPN was carried out in acetonitrile (polar) and toluene (nonpolar) solvents. Table $\mathbf{1}$ summarizes the photophysical and electrochemical properties of PPOCzPN, PPSCzPN, DiPPOCzPN and the reference emitter $2 \mathrm{CzPN}$ in polar solutions and doped films in PMMA (polymethyl methacrylate) as a polar host matrix, leading to the following observations. The phosphine chalcogenide-substituted emitters exhibited similar UV-visible absorption spectra to that of $2 \mathrm{CzPN}$ with three major bands observed at around $265 \mathrm{~nm}, 325 \mathrm{~nm}$, and $360 \mathrm{~nm}$ in both solvents (Figure 3a and 3d). The high-energy band near $265 \mathrm{~nm}$ is attributed to a LE transition of the carbazole moiety (see Figure S40) while the lowest energy bands near $325 \mathrm{~nm}$ and $360 \mathrm{~nm}$ are ascribed to ICT transitions in view of their low intensity. ${ }^{59}$ The ICT bands of the phosphine chalcogenide-substituted emitters are modestly blue-shifted by $380-1020 \mathrm{~cm}^{-1}(30-65 \mathrm{~nm})$ compared to the reference emitter $\mathbf{2 C z P N}$ due to the presence of the electron-withdrawing substituents. The presence of the second diphenylphosphine oxide group in DiPPOCzPN produced both a more intense absorption of the $\pi-\pi^{*}$ band at around $270 \mathrm{~nm}$ and the most blueshifted ICT band. The absorption spectra did not appreciably change between toluene and acetonitrile (see Figure 3a versus 3d).

The photoluminescence properties of PPOCzPN, PPSCzPN, DiPPOCzPN and 2CzPN were investigated in DCM and toluene, as neat films and as $10 \mathrm{wt} \%$ PMMA and $\mathrm{mCP}(1,3-b i s(N-$ carbazolyl)benzene) doped films, the latter matrix identified as a suitably high triplet-energy host that had previously been used with $\mathbf{2 C z P N}$. The photophysical properties are summarized in Tables $\mathbf{1}$ and $\mathbf{2}$ and the emission spectra and decay profiles of the emitters in doped PMMA and $\mathrm{mCP}$ thin films are shown in Figures $\mathbf{2 c}$ and $2 \mathbf{f}$. In DCM, the emission maxima shift to progressively higher energy across the series from 2CzPN to PPOCzPN, PPSCzPN, and DiPPOCzPN, at $532 \mathrm{~nm}, 498 \mathrm{~nm}, 501 \mathrm{~nm}$, and $465 \mathrm{~nm}$, respectively, reflecting the weakening of the donor. On the other hand, owing to its lower polarity, the emission maxima of these emitters are further blue-shifted in toluene compared to DCM, a reflection of their CT character. In $10 \mathrm{wt} \%$

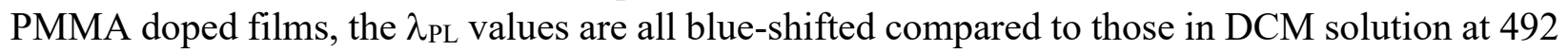
$\mathrm{nm}, 465 \mathrm{~nm}, 465 \mathrm{~nm}$, and $428 \mathrm{~nm}$, due the less polar nature of this medium (Figure 3b). In $10 \mathrm{wt} \%$ $\mathrm{mCP}$ doped films, the $\lambda_{\mathrm{PL}}$ values are surprisingly red-shifted and clustered closer to each other at $496 \mathrm{~nm}, 474 \mathrm{~nm}, 474 \mathrm{~nm}$, and $490 \mathrm{~nm}$ for 2CzPN, PPOCzPN, PPSCzPN, and DiPPOCzPN, respectively, in full consistency with the trends observed in toluene (Figure 3d). The photoluminescence quantum yields, $\Phi_{\mathrm{PL}}$, in DCM of PPOCzPN, PPSCzPN and DiPPOCzPN (47-61\%) are higher than that of $\mathbf{2 C z P N}(29 \%){ }^{79}$ The $\Phi_{\mathrm{PL}}$ values of the emitters are somewhat lower in PMMA doped films (ranging from 28-59\%), and are lower than that of $2 \mathbf{C z P N}$ (76\%) in the same medium. In mCP, the $\Phi_{\mathrm{PL}}$ is recovered with values of $72 \%, 73 \%$ and $56 \%$ for PPOCzPN, PPSCzPN and DiPPOCzPN, while that of $2 \mathrm{CzPN}$ is very similar to that seen in PMMA (77\%). A slightly higher $\Phi_{\mathrm{PL}}(89 \%)$ has been reported for $6 \mathrm{wt} \% \mathbf{2 C z P N}$ in co-evaporated mCP thin film. ${ }^{59}$

Figure 3c shows the time-resolved decay traces of the emitters in a PMMA matrix. The emission decay consisted of both prompt $\left(\tau_{\mathrm{p}}\right)$ nanosecond and delayed $\left(\tau_{\mathrm{d}}\right)$ microsecond fluorescence components. The prompt fluorescence decays of PPOCzPN, PPSCzPN and DiPPOCzPN were found to be bi-exponential with lifetimes, $\tau_{\mathrm{p}}$, ranging from 13-20 ns, similar to that of $2 \mathrm{CzPN}\left(\tau_{\mathrm{p}}=8,21 \mathrm{~ns}\right)$. The delayed fluorescence decay of the three emitters is triexponential in nature with average lifetime of $393 \mu \mathrm{s}, 416 \mu \mathrm{s}$, and $513 \mu \mathrm{s}$ for PPOCzPN, PPSCzPN and DiPPOCzPN, respectively, significantly longer than that of $2 \mathrm{CzPN}\left(\tau_{\mathrm{d}}=270 \mu \mathrm{s}\right)$. Figure $3 \mathbf{f}$ shows the emission decays in $\mathrm{mCP}$, which are similar to the decay profiles in the PMMA 
matrix. In this medium, the $\tau_{\mathrm{p}}$ ranges from 8-37 ns, longer than those measured in PMMA, and also longer than that of $\mathbf{2 C z P N}\left(\tau_{\mathrm{p}}=9,23 \mathrm{~ns}\right)$. The $\tau_{\mathrm{d}}$ values, on the other hand, for PPOCzPN, PPSCzPN and DiPPOCzPN in $10 \mathrm{wt} \% \mathrm{mCP}$ thin films were found to be $210 \mu \mathrm{s}, 206 \mu \mathrm{s}$, and 54 $\mu \mathrm{s}$, respectively, which are significantly shorter than those in PMMA and are also shorter than that of $2 \mathbf{C z P N}\left(\tau_{\mathrm{d}}=277 \mu \mathrm{s}\right)$. An average $\tau_{\mathrm{d}}$ of $273 \mu$ s has been reported previously for $6 \mathrm{wt} \% \mathbf{2 C z P N}$ in $\mathrm{mCP}$ thin film. ${ }^{59}$ The excited state lifetime of the emitters in doped films are summarized in Table 3. Altogether, in contrast to $\mathbf{2 C z P N}$, the present data for PPOCzPN, PPSCzPN and DiPPOCzPN emitters clearly demonstrate the high sensitivity of the emitter decay dynamics and emission energy towards the nature of the host medium.

Table 3. Excited state lifetimes of the emitters in doped films.

\begin{tabular}{|c|c|c|c|c|c|}
\hline \multirow[t]{2}{*}{ Emitter } & \multirow{2}{*}{\multicolumn{2}{|c|}{$10 \mathrm{wt} \%$ in PMMA films }} & \multicolumn{3}{|l|}{$10 \mathrm{wt} \%$ in $\mathrm{mCP}$ films } \\
\hline & & & $\tau_{p}(\%) / n s ; \tau_{d}(\%) / \mu s$ & $\begin{array}{l}\mathbf{S}_{1} / \\
\mathbf{e V}\end{array}$ & $\begin{array}{l}\mathbf{T}_{1} / \\
\mathbf{e V}\end{array}$ \\
\hline PPOCzPN & $\begin{array}{ccc}6.1 & (43), & 20.0 \\
9(3), & 146(22), & 1024(75)\end{array}$ & (57); & $\begin{array}{l}10.0(12), 37.2(88) ; 20(4), 90 \\
(18), 522(78)\end{array}$ & 2.96 & 2.77 \\
\hline PPSCzPN & $\begin{array}{llc}5.8 & (44), & 13.6 \\
5(0.5), & 174(10), & 1361(90\end{array}$ & $(56)$ & $\begin{array}{l}8.6, \quad(44), \quad 31.6 \quad(56) ; \\
23(4), 175(33), 422(62)\end{array}$ & 2.97 & 2.73 \\
\hline DiPPOCzPN & $\begin{array}{llr}6.7 & (40), & 14.1 \\
5(2), 69(6), 1176(92)\end{array}$ & $(60)$ & $\begin{array}{l}8.1 \quad(58), \quad 26.4 \quad(42) ; \\
0.5(76), 28(18), 136(6)\end{array}$ & 2.96 & 2.81 \\
\hline $2 \mathrm{CzPN}$ & $\begin{array}{lcc}8.3 & (15), & 21.1 \\
6(4), 124(22), & 680(72)\end{array}$ & (85); & $\begin{array}{l}9.9 \quad(29), \quad 23.2 \\
7(3), 134(23), 690(73)\end{array}$ & 2.90 & 2.67 \\
\hline
\end{tabular}

$\mathrm{S}_{1}=$ singlet state energy obtained from the onset of prompt fluorescence spectra measured at 77 $\mathrm{K}\left(\lambda_{\text {exc }}=360 \mathrm{~nm}\right.$, delay time: $1 \mathrm{~ns}$, time window: 1-100 ns). $\mathrm{T}_{1}=$ triplet state energy obtained from the onset of the phosphorescence spectra measured at $77 \mathrm{~K}\left(\lambda_{\text {exc }}=360 \mathrm{~nm}\right.$, delay time: 1 $\mathrm{ms}$, time window: 1-10 ms).

Temperature-dependent time-resolved emission decay measurements in $10 \mathrm{wt} \% \mathrm{mCP}$ doped thin films are shown in Figure S39. There is a clear thermal activation of the delayed fluorescence that is consistent with compounds emitting via a TADF mechanism, despite the different trends in comparison to the PMMA matrix. 

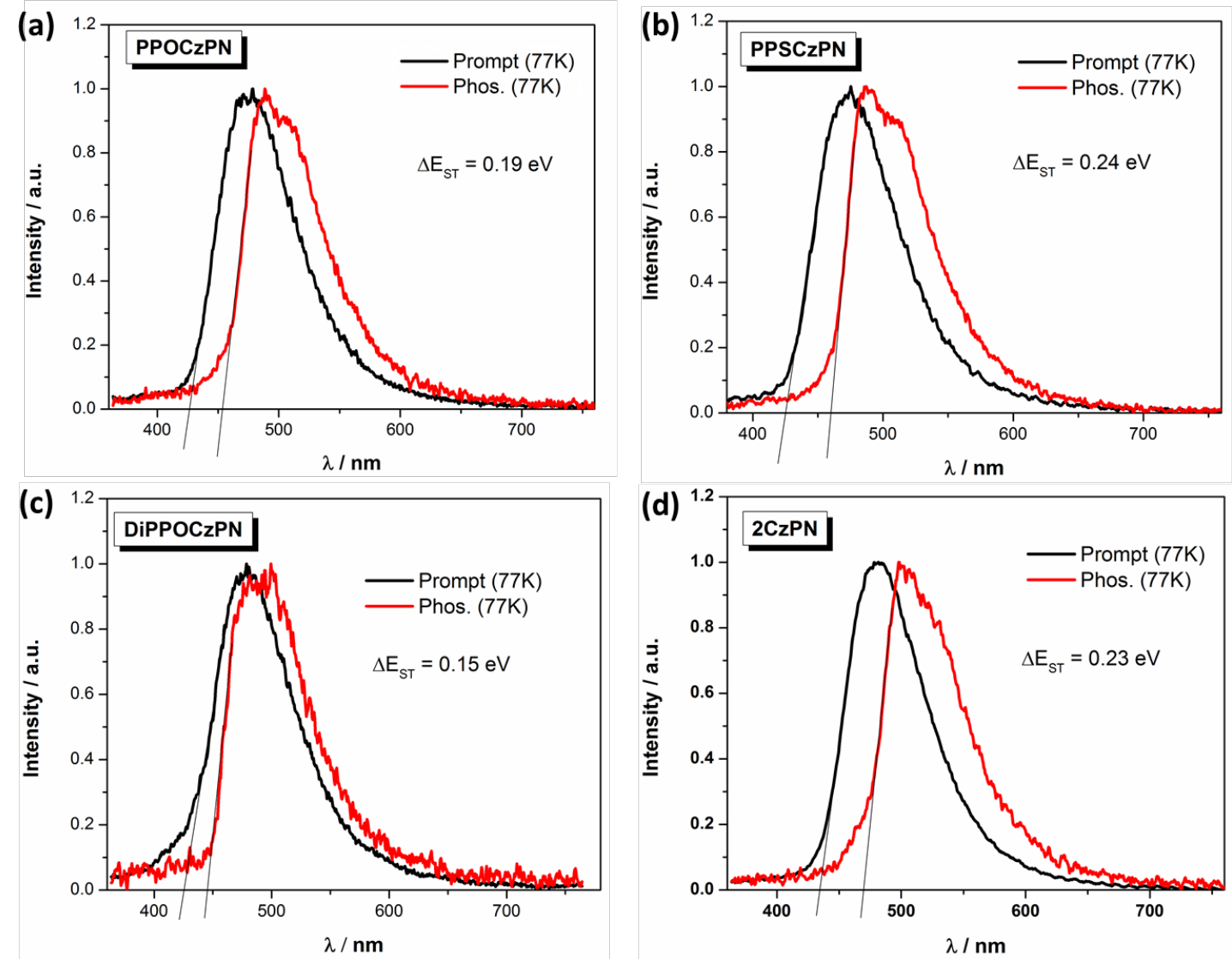

Figure 4. Prompt fluorescence (at $77 \mathrm{~K}$ ) and phosphorescence spectra (at $77 \mathrm{~K}$ ) of (a) PPOCzPN, (b) PPSCzPN, (c) DiPPOCzPN, and (d) 2CzPN, in $10 \mathrm{wt} \%$ doped $\mathrm{mCP}$ thin films $\left(\lambda_{\text {exc }}=360\right.$ $\mathrm{nm})$.

The singlet-triplet energy gaps $\left(\Delta E_{\mathrm{ST}}\right)$ were determined from the difference in energy of the onset of the prompt fluorescence and phosphorescence spectra in $10 \mathrm{wt} \% \mathrm{mCP}$ doped films measured at $77 \mathrm{~K}$ (Figure 4). The $\Delta E_{\mathrm{ST}}$ values for PPOCzPN, PPSCzPN DiPPOCzPN and 2CzPN were found to be $0.19 \mathrm{eV}, 0.24 \mathrm{eV}, 0.15 \mathrm{eV}$ and $0.23 \mathrm{eV}$, respectively. The experimentally

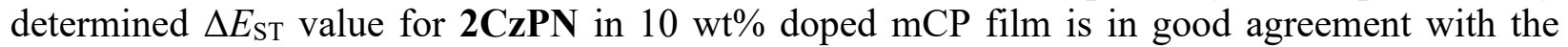
reported value of 0.21 in a $6 \mathrm{wt} \%$ doped $\mathrm{mCP}$ thin film. ${ }^{59,79}$ The smallest $\Delta E_{\mathrm{ST}}$ value $(0.15 \mathrm{eV}$ for DiPPOCzPN) is consistent with the observed $\tau_{\mathrm{d}}$, value of $54 \mu \mathrm{s}$, which is the shortest among these emitters. It is noteworthy that the TD-DFT calculated $\Delta E_{\mathrm{ST}}$ values in the gas phase are consistent with those experimentally determined in the apolar matrix, vide infra, Table 4 . The $\Delta E_{\mathrm{ST}}$ values determined for these chalcogenide-substituted $2 \mathbf{C z P N}$ derivatives are of similar magnitude to literature $2 \mathbf{C z P N}$ derivatives such as $\boldsymbol{\alpha - 2 C b P N}\left(\Delta E_{\mathrm{ST}}=0.28\right)$ and $\boldsymbol{\delta}-\mathbf{2 C b P N}\left(\Delta E_{\mathrm{ST}}=0.13\right)$ in 20 $\mathrm{wt} \% \mathrm{mCP}$ thin film, where $\alpha / \delta$-carboline was used as carbazole replacement to blue-shift the emission. ${ }^{60}$ 


\subsection{Theoretical modelling}

To better understand the differences between the PL behaviors observed in the polar DCM solvent or PMMA matrix versus the apolar toluene solvent or mCP matrix, our study has been complemented by quantum-chemical calculations. In line with our previous theoretical studies on TADF emitters, ${ }^{79,83}$ we have first optimized the ground-state geometry of the $\mathbf{2 C z P N}$ and its three derivatives in the gas phase at the Density Functional Theory (DFT) level using the PBE0 functional ${ }^{84}$ and a $6-31 \mathrm{G}(\mathrm{d}, \mathrm{p})$ basis set. ${ }^{85}$ In a second step, we have analyzed the nature of the frontier electronic levels of the compounds. Finally, we have described the lowest singlet and triplet excited states of the emitters by performing TD-DFT calculations within the Tamm-Dancoff approximation, ${ }^{86}$ employing the same functional and basis set.

In the case of the isolated PPOCzPN and PPSCzPN compounds, we have obtained two different low-energy conformers varying by the amplitude of the torsion angles $\phi$ between the carbazole and phthalonitrile units (see Figure S40). For PPOCzPN, rotamer 2 with $\phi_{1}=134.3^{\circ}$ and $\phi_{2}=-69.2^{\circ}$ is more stable by $0.29 \mathrm{eV}$ compared to the other rotamer with $\phi_{1}=-64.8^{\circ}$ and $\phi_{2}=-$ $65.9^{\circ}$; in the case of PPSCzPN, there is a much larger difference of $1.33 \mathrm{eV}$ between the most stable rotamer (with $\phi_{1}=133.2^{\circ}$ and $\phi_{2}=-69.5^{\circ}$ ) compared to the second rotamer (with $\phi_{1}=-65.8^{\circ}$ and $\phi_{2}=-64.6^{\circ}$ ). In view of the large energy difference between the two rotamers (compared to the thermal energy, kT), their co-existence in solution most probably arises from their synthesis, rather than from thermal conversion following synthesis. We will discuss hereafter the properties of the most stable rotamer; for sake of completeness, the very similar properties computed for the second rotamer in the gas phase are collected in the Supporting Information (Figures S40-S41 and Tables S2 and S4). In contrast, we only found a single stable conformer for $\mathbf{2 C z P N}$ and DiPPOCzPN with the same torsion angles for both carbazole substituents $\left(\phi_{1}=\phi_{2}=58.9^{\circ}\right.$ for 2CzPN and $\phi_{1}=\phi_{2}=65.7^{\circ}$ for DiPPOCzPN).

Table 4. Calculated HOMO and LUMO energies of PPOCzPN, PPSCzPN, DiPPOCzPN and $\mathbf{2 C z P N}$ in gas phase versus MeCN.

\begin{tabular}{|c|c|c|c|c|c|c|}
\hline & \multicolumn{3}{|c|}{ In gas phase } & \multicolumn{3}{|c|}{ In $\mathrm{MeCN}$} \\
\hline & $\begin{array}{l}\text { HOMO } \\
\text { /eV }\end{array}$ & $\begin{array}{l}\text { LUMO } \\
\text { /eV }\end{array}$ & $\begin{array}{l}\Delta \mathbf{E} \\
/ \mathbf{e V}\end{array}$ & $\begin{array}{l}\text { HOMO } \\
\text { /eV }\end{array}$ & $\begin{array}{l}\text { LUMO } \\
/ \mathrm{eV}\end{array}$ & $\begin{array}{l}\Delta \mathbf{E} \\
/ \mathbf{e V}\end{array}$ \\
\hline PPOCzPN & -6.05 & -2.52 & 3.53 & -6.06 & -2.48 & 3.58 \\
\hline PPSCzPN & -5.98 & -2.58 & 3.40 & -6.13 & -2.50 & 3.63 \\
\hline DiPPOCzPN & -6.42 & -2.73 & 3.69 & -6.37 & -2.57 & 3.80 \\
\hline $2 \mathrm{CzPN}$ & -6.11 & -2.41 & 3.70 & -6.04 & -2.39 & 3.65 \\
\hline
\end{tabular}

Table 4 collects the HOMO and LUMO energies of the four emitters calculated in the gas phase as well as in acetonitrile by modelling the solvent as a dielectric continuum using the Polarizable Continuum Model (PCM) ${ }^{87}$, as implemented in Gaussian 16-A03; ${ }^{88}$ in the latter case, the geometry is fully reoptimized within the PCM model, although this hardly changes the gas phase geometry. In acetonitrile, the HOMO is progressively stabilized going from $\mathbf{2 C z P N}$ to 
PPOCzPN to PPSCzPN and DiPPOCzPN, which is generally consistent with the trends observed in the experimental electrochemistry data in DCM (Table 1) and acetonitrile (Figure S36). On the other hand, the calculated LUMO energies vary to a lesser extent both according to the calculations and electrochemical measurements, as expected by the fact that the LUMO is localized on the phthalonitrile acceptor while the varying substituents are added on the donor fragment. Strikingly, the trends appear to be quite different when computing the frontier orbital energies in the gas phase, which is expected to reflect the properties in a non-polar medium such as toluene or the $\mathrm{mCP}$ matrix. The major difference is that the HOMO is not stabilized but in fact slightly destabilized when going from 2CzPN to PPOCzPN or PPSCzPN, although it is stabilized going from 2CzPN to DiPPOCzPN.

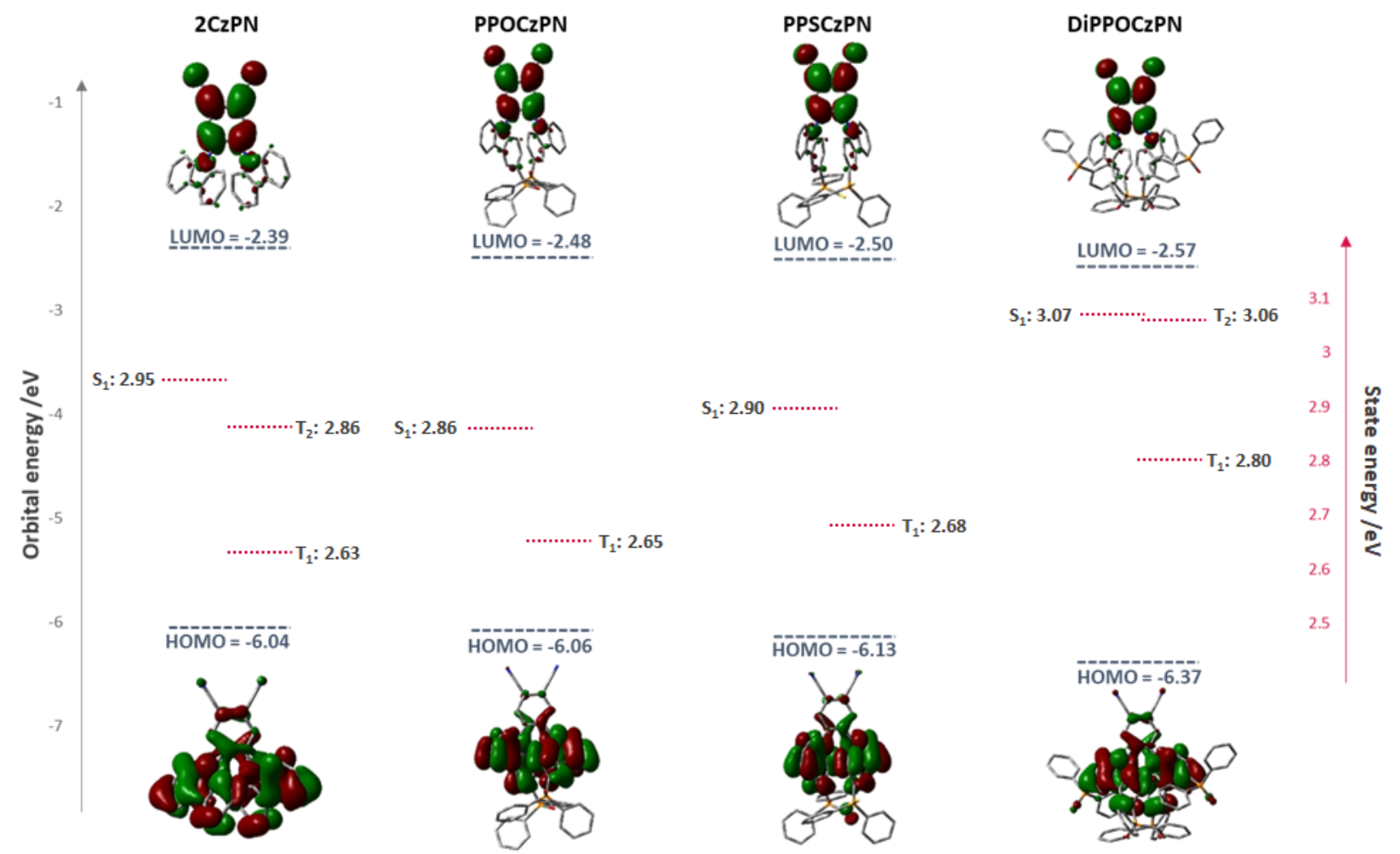

Figure 5. HOMO and LUMO energies of the four compounds in their most stable geometries together with the energy of the lowest singlet and triplet excited states, as calculated in acetonitrile.

The shape and energies of the orbitals in acetonitrile are displayed in Figure 5 (and Figure S41 for the less stable rotamers of PPOCzPN and PPSCzPN). As expected, the HOMOs are mainly localized on the carbazole moieties, and the LUMOs are strongly localized on the phthalonitrile. In the monosubstituted compounds, the HOMO displays a small amount of electron density on the phosphine chalcogenide units, thus limiting the role played by mesomeric effects in defining the actual HOMO energies. The HOMO level is both the most delocalized in DiPPOCzPN and the most stabilized, which is also evidenced by its electrochemistry (Figures 2 and S36). The shift of the HOMO among the compounds is a subtle interplay mostly between the inductively electron-withdrawing effects associated with the phosphine chalcogenide groups and 
electrostatic interactions between the polar phosphine chalcogenide and cyano moieties, which are expected to be further tuned by changes in the medium polarity. Altogether, the HOMO-LUMO gap in the gas phase is red shifted by $0.2-0.3 \mathrm{eV}$ going from 2CzPN to PPOCzPN and PPSCzPN while it remains unchanged for DiPPOCzPN. In contrast, the electronic bandgaps of 2CzPN, PPOCzPN, PPSCzPN are quite similar in MeCN and larger by $\sim 0.2 \mathrm{eV}$ for DiPPOCzPN, thus demonstrating the strong impact of the medium polarity on the electronic structure of the compounds and by extension on the charge-transfer state energies, as evidenced at the experimental level.

When coupled to a time-dependent (TD) formalism, DFT calculations can also give access to the excited-state photophysical properties of the emitters. We will describe below the excited state energies and associated oscillator strength $(f)$ calculated in acetonitrile as well as the energy difference between $T_{1}$ and $S_{1}$ (Table 5) for vertical excitation processes (i.e. in the ground-state geometry). We will not address here at the theoretical level the changes in the emission properties as a function of the medium polarity evidenced experimentally, since a proper analysis would require a time-consuming optimization of the $S_{1}$ state (both in gas phase and in a polar medium described with PCM) which is often impeded by convergence problems and might require the use of another functional. ${ }^{84}$ PPOCzPN and PPSCzPN exhibit a slightly red-shifted $\mathrm{S}_{1}$ state compared to $2 \mathrm{CzPN}$ (blue-shifted in the case of DiPPOCzPN), accompanied by a reduction in the oscillator strength, as well as slightly smaller $\Delta E_{\mathrm{ST}}$ values. The calculated $\Delta E_{\mathrm{ST}}$ values of the emitters in the toluene are all around or above $0.2 \mathrm{eV}$, in good consistency with those experimentally obtained in toluene (Table 2); there is a very good quantitative agreement for $\mathbf{2} \mathbf{C z N}(0.31 \mathrm{eV}$ in theory versus $0.36 \mathrm{eV}$ in toluene). These energies are marginally affected when performing the calculations in toluene or in acetonitrile (Table S4). Strikingly, the experimental $\Delta E_{\mathrm{ST}}$ values are systematically reduced in the $\mathrm{mCP}$ matrix, most likely due in part to changes in the conformations of the emitters in these thin films. Both $\mathbf{2 C z P N}$ and DiPPOCzPN have a second triplet excited state $\mathrm{T}_{2}$ that lies below $\mathrm{S}_{1}$, thus providing a conduit to facilitate reverse intersystem crossing process. ${ }^{74,89}$ The $_{1}$ and $\mathrm{S}_{1}$ excited states are predominantly characterized by a HOMO-LUMO transition and hence both display a significant ICT character.

Table 5. Excitation energies, $\Delta \mathrm{E}_{\mathrm{ST}}$, oscillator strengths, and main component of the excitations in terms of one-electron transitions, as calculated in acetonitrile.

\begin{tabular}{c|ccccc}
\hline Compound & States & $\begin{array}{c}\text { Energy } \\
\text { /eV }\end{array}$ & $\boldsymbol{f}$ & $\begin{array}{c}\text { Main component of the } \\
\text { excitation }\end{array}$ & $\boldsymbol{\Phi}_{\mathbf{s}}$ \\
\hline PPOCzPN & $\mathrm{T}_{1}$ & 2.65 & & HOMO $\rightarrow$ LUMO (76.5\%) & 0.60 \\
& $\mathrm{~S}_{1}$ & 2.86 & 0.028 & HOMO $\rightarrow$ LUMO (97.7\%) & 0.35 \\
\hline PPSCzPN & $\mathrm{EE}_{\mathbf{S T}}$ & $\mathbf{0 . 2 1}$ & & & \\
\hline
\end{tabular}




\begin{tabular}{|c|c|c|c|c|c|}
\hline & $\begin{array}{c}\mathrm{S}_{1} \\
\Delta \mathbf{E}_{\mathbf{S T}}\end{array}$ & $\begin{array}{l}2.90 \\
\mathbf{0 . 2 2}\end{array}$ & 0.032 & HOMO $\rightarrow$ LUMO (97.9\%) & 0.37 \\
\hline \multirow[t]{4}{*}{ DiPPOCzPN } & $\mathrm{T}_{1}$ & 2.80 & & HOMO $\rightarrow$ LUMO (82.1\%) & 0.66 \\
\hline & $\mathrm{T}_{2}$ & 3.06 & & & 0.50 \\
\hline & $\mathrm{S}_{1}$ & 3.07 & 0.045 & HOMO $\rightarrow$ LUMO (98.3\%) & 0.39 \\
\hline & $\Delta \mathbf{E}_{\mathbf{S T}}$ & 0.27 & & & \\
\hline \multirow[t]{4}{*}{$2 \mathrm{CzPN}$} & $\mathrm{T}_{1}$ & 2.63 & & HOMO $\rightarrow$ LUMO $(87.8 \%)$ & 0.66 \\
\hline & $\mathrm{T}_{2}$ & 2.86 & & & 0.53 \\
\hline & $\mathrm{S}_{1}$ & 2.95 & 0.100 & HOMO $\rightarrow$ LUMO (98.4\%) & 0.43 \\
\hline & $\Delta \mathbf{E}_{\mathbf{S T}}$ & 0.32 & & & \\
\hline
\end{tabular}

The absorption spectra simulated in the gas phase (Figure $\mathbf{S 4 3}$ ) predict similar energy for the lowest absorption peaks (around $350 \mathrm{~nm}$ and $400 \mathrm{~nm}$ ) for the four compounds; the same trends are also observed for the experimental spectra recorded both in $\mathrm{MeCN}$ and toluene (Figures 1a and 1d). Note that the lowest absorption peak around $400 \mathrm{~nm}$ is systematically associated with higherlying excited states while the lowest excited state $S_{1}$ gives rise to the shoulder on the low energy side. This state is mostly described by a HOMO to LUMO excitation. Analysis of the hole and electron densities (see typical plots for the gas phase in Figure S42) obtained within the attachment/detachment formalism points to a similar charge transfer character in the $\mathrm{S}_{1}$ state of the four compounds in acetonitrile or in gas phase (Table 5); the overlap index $\phi s$, ranging from 1 for a pure locally excited state (LE) to 0 for a pure charge-transfer (CT) state, is around 0.4 in all cases, i.e., an indication of a hybrid state with significant LE and CT character. The $\mathrm{T}_{1}$ state exhibits systematically a higher $\phi_{S}$ index pointing to a more local excited state. This change in character between the $S_{1}$ and $T_{1}\left(T_{2}\right)$ states ensures non-vanishing spin-orbit coupling based on El Sayed's rules and opens up the RISC channel.

\subsection{Electrochemiluminescence via the Annihilation Pathway}



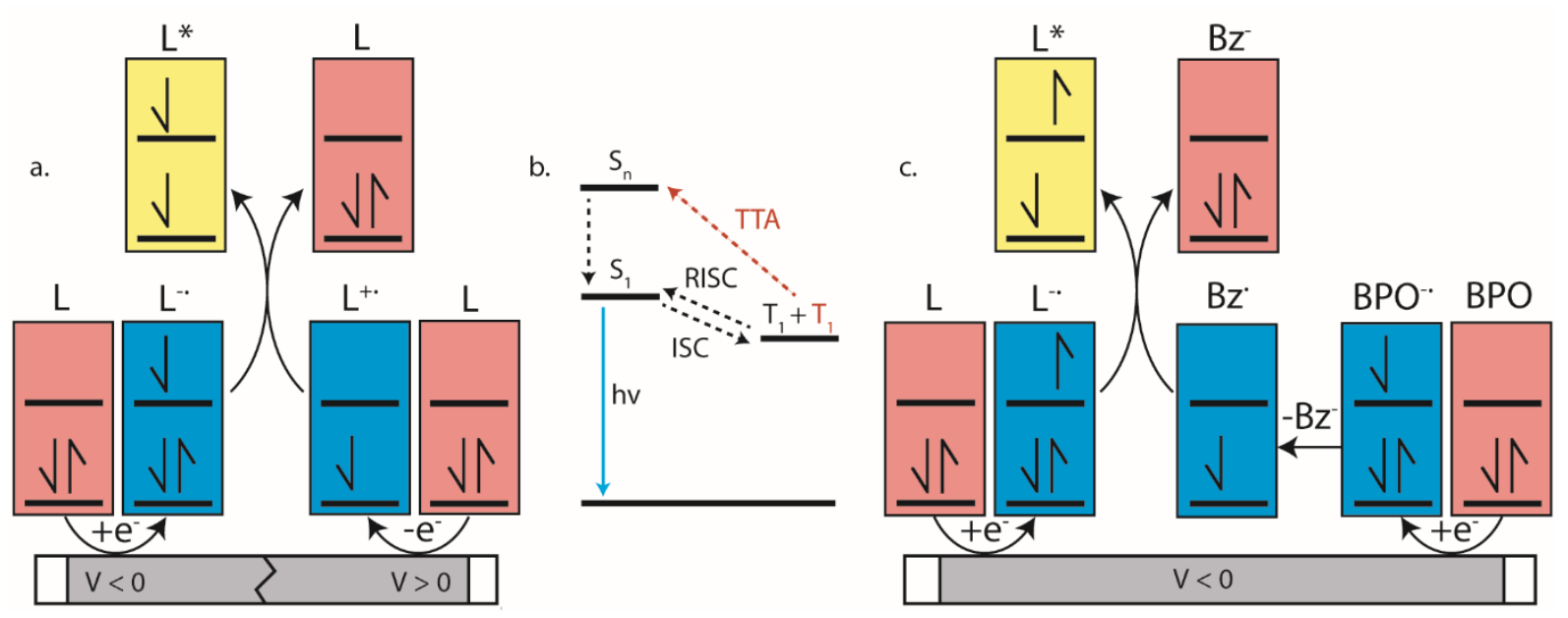

Figure 6.(a) Annihilation and (c) BPO co-reactant mechanisms for ECL emission. L, Bz and BPO stand for luminophore, benzoate and benzoyl peroxide, respectively. This scheme is not energy accurate and both triplet and singlet energy states can be accessed from either co-reactant or annihilation mechanisms. (b) Jablonski diagram illustrating intersystem crossing, reverse intersystem crossing, triplet-triplet annihilation (TTA) mechanisms and singlet emission.

ECL is luminescence generated when electrochemically-generated radical cations (hole) and radical anions (electron) undergo an electron transfer to create an excited state (exciton), which can release its energy as light (Figure 6a). The behavior of this luminescence can describe radical stability, reactivity and general emission mechanisms of luminophores at concentrations that are typically greater than that in solutions used in photoluminescence studies. Radical cations and anions are produced in solution when the species are oxidized and reduced, respectively in the vicinity of the same working electrode. If these two species collide, an electron transfer can happen from the HOMO (or LUMO) of a radical anion to the HOMO (or LUMO) of a radical cation, potentially creating an exciton that can subsequently emit light in the form of ECL. This mechanism is not limited to the generation of triplets as illustrated in Figure 6a, but also can form systematically singlets in the case of an annihilation mechanism, in an analogous manner to exciton generation in an organic light-emitting diode.

To electrogenerate an excited state, the electronic gap of the redox reactions $\left(\mathrm{EE}_{\mathrm{g}}\right)$ must be greater than the photoluminescence emission peak energy $\left(E_{g}\right)$. Assuming an elementary charge of 1 , one must ensure that:

$$
E g \leq E^{\circ}\left(\frac{R^{\circ+^{\prime}}}{R^{\prime}}\right)-E^{\circ}\left(\frac{R}{R^{\circ-}}\right)=E E g
$$

For an ECL annihilation system, R' and R are the same species. However, R' can become a radical while $\mathrm{R}$ remains the luminophore in an oxidative-reduction ECL co-reactant system or R can become an oxidant while $\mathrm{R}^{\prime}$ remains a luminophore in a reductive-oxidation ECL co-reactant system. Using Eq. 1, the electronic gap for PPOCzPN, PPSCzPN and DiPPOCzPN in DCM are measured to be $2.73,2.98$ and $3.19 \mathrm{eV}$, respectively, from the redox potentials in differential pulse voltammograms (Figure S35). From the prompt fluorescence spectra in DCM in Figure 4, the optical gap for PPOCzPN, PPSCzPN and DiPPOCzPN are 2.49, 2.47 and $2.66 \mathrm{eV}$, respectively. 
As expected, the energy provided by the redox reactions for all compounds is sufficient to generate both singlet and triplet excited states. The blue curves in Figure 2a-c demonstrate ECL generated during scan cycles between redox reaction potentials for PPOCzPN, PPSCzPN and DiPPOCzPN. DiPPOCzPN shows a maximum ECL intensity of $60 \mathrm{nA}$, while PPOCzPN and PPSCzPN display weak emissions up to $1 \mathrm{nA}$. This indicates that the radical cation is sufficiently stable to survive in solution until the radical anion is created, and the two species collide. DiPPOCzPN $^{\cdot+}$ is the most stable radical ion and can produce highly emissive ECL in the annihilation pathway. It also appears that the radical anion is not as stable as the radical cation for all species, because no ECL is seen upon first reduction of the species followed by compound oxidation, even after multiple cycles.

Figure 2d-f shows ECL-time curves during pulsing between $0.1 \mathrm{~V}$ more anodic than the oxidation and $0.1 \mathrm{~V}$ more cathodic than reduction peak potentials, respectively. PPOCzPN, PPSCzPN and DiPPOCzPN yielded annihilation ECL maxima at 700, 1100 and $17500 \mathrm{nA}$, respectively. In pulsing experiments, the time between the generation of both radical species in solution is shorter than that in potential cycling, allowing the lifetime of the radical not to be a significant issue and the annihilation ECL to be enhanced. The pulsing annihilation efficiency was determined by Eq. 2:

$$
\Phi_{\mathrm{ECL}}=\frac{\left(\frac{\int \mathrm{ECL} d \mathrm{~d}}{\int \text { Current dt }}\right)_{x}}{\left(\frac{\int \mathrm{ECLdt}}{\int \text { Current dt }}\right)_{s t}} \times 100 \%
$$

where $x$ and $s t$ stand for the luminophore and $\left[\mathrm{Ru}(\mathrm{bpy})_{3}\right]^{2+}$ under the same conditions, respectively. These ECL efficiencies were determined to be 1,1 and $10 \%$ relative to $\left[\mathrm{Ru}(\mathrm{bpy})_{3}\right]^{2+}$, for PPOCzPN, PPSCzPN and DiPPOCzPN, respectively (Table 6). Interestingly, 2CzPN showed no emissions in the annihilation pathway as seen in Figure S38a-b. DiPPOCzPN continued to have the highest ECL maxima and also had the most efficient emission; interestingly, the structurally similar PPOCzPN had the lowest efficiency. Most emission was observed when a negative potential was applied, further providing evidence for the higher stability of the radical cation over the radical anion. In fact, PPOCzPN is the only species to display emission when a positive potential was applied or when radical cations were actively being produced in pulsing experiments (2d-e), indicating a relatively high radical anion reactivity, or a relatively lower radical cation reactivity than the other compounds. For optoelectronic applications, ECL efficiency, ECL maxima, radical stability and radical reactivity are all important parameters to consider. Inequalities between radical cation and radical anion stabilities and reactivities can cause excess charge buildup in OLEDs, leading to fast device degradation. High ECL maxima and efficiencies indicate efficient charge transfers as well as limited non-radiative decay pathways. Therefore, PPOCzPN appears desirable for optoelectronic applications because of relatively more stable and reactive radical ion species, although it has low ECL maxima and efficiency.

Table 6. Summary of ECL data.

\begin{tabular}{lllll}
\hline & PPOCzPN & PPSCzPN & DiPPOCzPN & {$\left[\mathbf{R u}(\mathbf{b p y})_{3}\right]^{2+}$} \\
\hline $\mathbf{E E}_{\mathbf{g}}(\mathbf{e V})^{a}$ & 2.73 & 2.98 & 3.19 & 2.55
\end{tabular}




\begin{tabular}{|c|c|c|c|c|}
\hline Pulsing ECL ${\text { onset delay }(\mathrm{ms})^{b}}^{b}$ & 7 & 14 & 11 & 0 \\
\hline Pulsing ECL $L_{\max }$ delay (ms) ${ }^{b}$ & 11 & 19 & 24 & 4 \\
\hline Pulsing ECL efficiency $(\%)^{c}$ & 1 & 1 & 10 & 100 \\
\hline Max pulsing ECL (nA) & 700 & 1,150 & 17,500 & 70,000 \\
\hline Annihilation ECL $\lambda_{\max }(\mathrm{nm})$ & 520 & 500 & 475 & 620 \\
\hline $\mathrm{EE}_{\mathrm{g}} \mathrm{BPO}(\mathrm{eV})^{d}$ & 3.01 & 3.48 & 3.39 & 3.04 \\
\hline BPO ECL efficiency $(\%)^{c}$ & 17 & 6 & 1 & 100 \\
\hline Max BPO ECL (nA) & 3,200 & 1,280 & 80 & 26,500 \\
\hline BPO ECL $\lambda_{\max }(\mathbf{n m})$ & 520 & 500 & 545 & 620 \\
\hline
\end{tabular}

\begin{abstract}
${ }^{a}$ Electronic gaps $\left(\mathrm{EEg}_{\mathrm{g}}\right)$ were obtained using Eq. 1 and the differential pulse voltammograms of the compounds in Figure S36. ${ }^{b}$ These values are averages from at least 40 pulses with individual conditions described in Figure 2. ${ }^{c}$ Obtained from Eq. 2 relative to $\left[\mathrm{Ru}(\mathrm{bpy})_{3}\right]^{2+}$ at the same concentration, electrolyte and concentration of co-reactant if applicable. ${ }^{d}$ Obtained from Eq. 1 but with substituting $\mathrm{E}^{\circ}\left(\mathrm{R}^{\cdot+1} / \mathrm{R}^{\prime}\right)$ with the oxidative power of $\mathrm{BPO}$ from ref. ${ }^{90}$
\end{abstract}

After several pulses, the emission intensity of DiPPOCzPN decreases by over $75 \%$, whereas PPOCzPN and PPSCzPN remain relatively stable over the same time (Figure S45). It appears that DiPPOCzPN may form side products during the annihilation pulsed ECL experiment that influence the generation of excited species. This fast decay may also be caused by a charge imbalance where negatively or positively charged species that accumulate over time interfere with the exciton states, similar to exciton-polaron quenching of excited states. ${ }^{91}$

\title{
Average ECL Onset Times
}

These experiments reveal for the first time (Figure 2d-f) that there is a delay in the ECL onset times (ECL onset $_{\text {) }}$ from donor-acceptor TADF compounds; PPOCzPN, PPSCzPN and DiPPOCzPN had 7 (26 pulses measured), 14 (36 pulses measured) and $11 \mathrm{~ms}$ (37 pulses measured) initial ECL delays, respectively. The data are summarized in Table 6 and zoom-in ECLtime curves of a single pulse of each compound are shown in Figure S46. This ECL emission delay is currently unexplained in the literature. Although singlet and triplet ECL excitation pathways are energetically sufficient and possible, the significant delay observed in ECL onset times indicates a long emission mechanism. We believe this long emission time cannot be explained solely by triplet-triplet annihilation (TTA), luminescence or diffusion of the species, given the absence of such observed delays for $\left[\mathrm{Ru}(\mathrm{bpy})_{3}\right]^{2+}$ (vide infra), and so another factor must be considered.

For time comparisons of diffusion properties and emissions, the well-studied commercial phosphorescent ECL emitter $\left[\mathrm{Ru}(\mathrm{bpy})_{3}\right]^{2+},{ }^{92-95}$ was tested under the same conditions (Figure S46) 
and showed no observed delay (47 pulses measured). A well-studied organic compound with a known diffusion coefficient is diphenylanthracene (DPA) $\left(D_{\mathrm{ACN}}=0.20 \times 10^{-6} \mathrm{~cm}^{2} \mathrm{~s}^{-1}\right)^{96}$, which shows an order of magnitude difference between its diffusion coefficient and that of $\left[\mathrm{Ru}(\mathrm{bpy})_{3}\right]^{2+}$ $\left(\mathrm{D}_{\mathrm{ACN}}=5.8 \times 10^{-6} \mathrm{~cm}^{2} \mathrm{~s}^{-1}\right) \cdot{ }^{97}$ If we assume that DPA and these $2 \mathrm{CzPN}$ derivatives have similar magnitudes of diffusion coefficient in DCM, from a comparison of their initial emission delays (7 and $0 \mathrm{~ms}$ for PPOCzPN and $\left[\mathrm{Ru}(\mathrm{bpy})_{3}\right]^{2+}$, respectively), differences in diffusion rates can be ruled out as a cause of emission delays in the $2 \mathbf{C z P N}$ derivatives. We believe diffusion delays are on the time scale of microseconds and cannot explain the observed time delay shown in Figure 2d-f.

A TTA mechanism has been used to explain millisecond photoluminescence lifetimes for phenanthrene and may explain the delay of the onset of the ECL seen here. ${ }^{98}$ Also, the competition for triplet exciton depopulation by TTA, RISC and non-radiative pathways has been studied by Grüne et al. in a m-MTDATA:3TPYMB exciplex TADF OLED to estimate that TTA was responsible for $50 \%$ of triplet depopulation, which effectively extended the emission decay time. ${ }^{99}$ However, Grüne et al. excited their sample for $4 \mathrm{~ms}$ at a specific current density and did not see any delay in the onset of emission. TTA has been observed in carbazolyl phthalonitrile derivatives where there is an observed blue-shifting in the solid-state PL as well as serious efficiency roll-off in the OLEDs, ${ }^{100-101}$ but to the best of our knowledge it has not been demonstrated before by ECL in $\mathbf{2 C z P N}$ derivatives. TTA can be simplified to two triplet excitons $\left(\mathrm{T}_{1}\right)$ combining to form a singlet exciton $\left(\mathrm{S}_{\mathrm{n}}\right)$ seen in Figure $\mathbf{6 b}$. The system in the $\mathrm{S}_{\mathrm{n}}$ state then relaxes to the $\mathrm{S}_{1}$ state by internal conversion according to Kasha's law, which can then emit light. TTA is also a common tetramolecular process in ECL where the radical cation of $N, N, N^{\prime}, N^{\prime}$-tetramethyl- $p$ phenylenediamine $\left(\mathrm{TMPD}^{\circ+}\right.$ ) and the radical anion of DPA (DPA $\left.{ }^{--}\right)$reside as a well-studied purely TTA-emitting ECL system. ${ }^{90,102-104}$ This $\mathrm{TMPD}^{\cdot+} / \mathrm{DPA}^{--}$system can only generate $\mathrm{T}_{1}$ from charge transfer reactions and not $\mathrm{S}_{1}$ as dictated by Eq. 1. However, this TTA mechanism has never been accompanied by an observed induction time either in ECL or OLED prior to observed emission.

To the best of our knowledge, there has been only one study of emission delay after excitation in an ECL system, where the ECL of DPA was found to have a delay on the order of $100 \mu$ s. $^{105}$ This delay was attributed to uncompensated solution resistance. In comparison, our experiment also has a platinum working electrode with the same supporting electrolyte (TBAP) at a similar concentration, but the delay we determined is a few orders of magnitude larger than what Rosenmund et al. observed. Solution resistance is likely not the culprit of our much delayed emission. Interestingly, an organic long-persistent photoluminescence (OLPL) phenomenon was recently discovered by Kabe et al. in a system comprising a phosphine oxide-containing TADF acceptor (2,8-bis(diphenylphosphoryl)dibenzo-[b,d]thiophene) (PPT)) and $N, N, N^{\prime}, N^{\prime}$ tettramethylbenzidine (TMB) as a donor molecule. ${ }^{106}$ More recently, this OLPL system has been used in an OLED to achieve organic long-persistent electroluminescence (OLEL). ${ }^{107-108}$ Unfortunately, solution and solid state emission delays of the system were not investigated on time scales smaller than $1 \mathrm{~s}$, thereby limiting comparisons with the $2 \mathbf{C z P N}$ derivatives. However, OLPL and OLEL are both emitted from an exciplex state and therefore the emission was found to be both concentration dependent and layer-thickness dependent. This exciplex emission was also found to have thermal activation processes and thermoluminescence in films. ${ }^{108} \mathrm{Li}$ et al. reported that the TADF emitter, CzPhAP, did not show any delay in the onset of emission but did show $900 \mathrm{~s}$ of persistent emission after $60 \mathrm{~s}$ of laser excitation, when cast in a PMMA film. ${ }^{109}$ These unusually long emission times were ascribed to slow charge recombination rates 
of the luminophore in the thick film, leading to organic long-persistent photoluminescence (OLPL). We believe that the delay in the onset of the ECL emission seen in Figure $2 \mathbf{d - f}$ may be best explained by a heretofore previously unreported organic long-persistent electrochemiluminescence (OLECL), analogous to the works of Kabe et al.

\section{Average ECL Rising Times to Maximum}

The average times from the onset of ECL to the ECL emission maximum after a pulse for PPOCzPN, PPSCzPN and DiPPOCzPN were found to be 11 (78 pulses measured), 19 (37 pulses measured) and $24 \mathrm{~ms}$ (77 pulses measured), respectively (Figure 2d-f and Table 6). Again, for comparison, $\left[\mathrm{Ru}(\mathrm{bpy})_{3}\right]^{2+}$ was analyzed using the same ECL pulsing method and this compound shows a rise time of $4 \mathrm{~ms}$ (158 pulses measured, Figure S47). Notably, the magnitude of the delay in reaching the maximum ECL emission intensity (Table 6) correlates with the trends in $\Delta E_{\mathrm{ST}}$ values in toluene (Table 2), where PPOCzPN has the smallest $\Delta E_{\mathrm{ST}}$ at $0.20 \mathrm{eV}$, and PPSCzPN and DiPPOCzPN have the largest $\Delta E_{\mathrm{ST}}$ at 0.42 and $0.43 \mathrm{eV}$, respectively (Figure 7). Based on this relationship, we can confidently rule out TTA as a dominant emission mechanism. Interestingly, the OLPL TADF emitter CzPhAP mentioned earlier had a similarly large $\Delta E_{\mathrm{ST}}$ of 0.18 compared with PPOCzPN (Table 2).

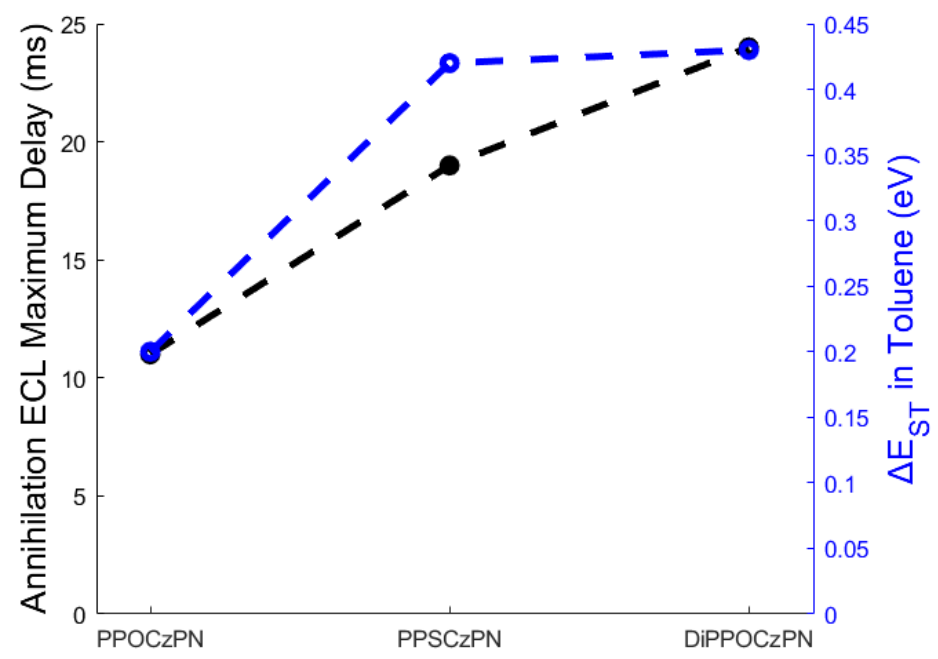

Figure 7. A relationship between the $\Delta E_{\mathrm{ST}}$ in toluene and the annihilation ECL maximum delay of the 2CzPN derivatives in this study.

\section{Average long emission decay time}

The average long emission decay time after switching off pulses was measured to find how long it took ECL emission to return to $0 \mathrm{nA}$ after the potential is switched off (Figures $\mathbf{4 d - f}$ and S47). These long emission decay times were found to be 0,6 , and $6 \mathrm{~ms}$ for PPOCzPN, PPSCzPN and DiPPOCzPN, respectively. Expectedly, the same trends for the delay to the onset of ECL and the time required from the ECL onset to reach a maximum ECL intensity were observed. However, the ECL decay times were roughly half the magnitude of the ECL onset times. By contrast, $\left[\mathrm{Ru}(\mathrm{bpy})_{3}\right]^{2+}$ shows instantaneous ECL emission during both cathodic and anodic pulses (Figure S47), and rapid decay of the ECL emission, which could not be quantified. 


\subsection{ECL of BPO Co-reactant Systems}
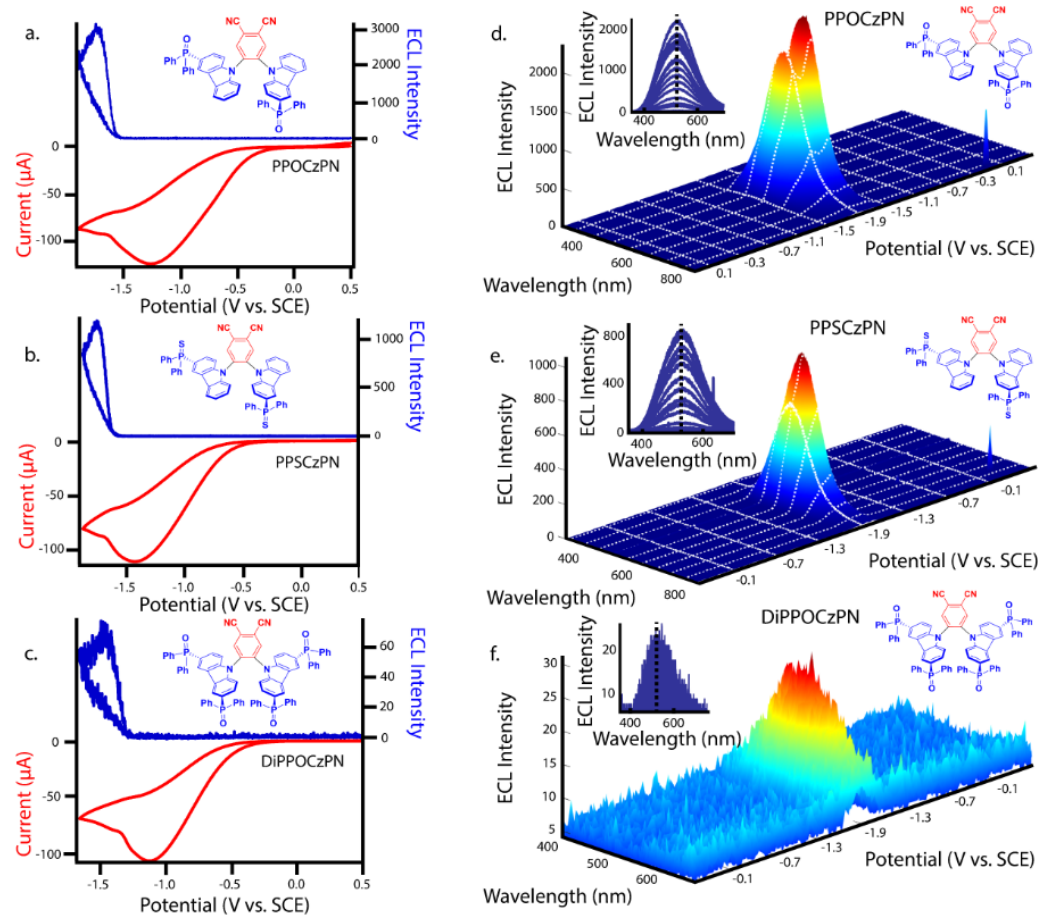

Figure 8. (a-c) CVs (red) along with ECL-voltage curves (blue) during potential pulsing at a pulsing frequency of $10 \mathrm{~Hz}$ for PPOCzPN, PPSCzPN and DiPPOCzPN, respectively, all with 10 mM BPO was added as a co-reactant. (d-f) Spooling ECL spectroscopy of the corresponding systems described in Figures 6a-c. Insets of Figures 6d-f represent respective stacked spooling ECL spectra. The purple color of these insets was arbitrarily chosen but Figure $\mathbf{S 4 8}$ displays the observable colour of each recorded spectrum.

BPO was added as a co-reactant to the electrochemical systems described in Figure $\mathbf{2}$ and the potential was scanned in the cathodic direction. This co-reactant pathway is described in Figure 6c. Around $-0.45 \mathrm{~V}$ vs. SCE, BPO is reduced and ultimately decomposes into a benzoate radical (Scheme 3c). When PPOCzPN, PPSCzPN and DiPPOCzPN are subsequently reduced to form PPOCzPN $^{\bullet}$, PPSCzPN*- and DiPPOCzPN*-, the benzoate radical produced earlier can abstract an electron from the HOMOs of the reduced TADF compounds to produce excited states PPOCzPN*, PPSCzPN* and DiPPOCzPN*, respectively. These excited-state compounds then emit ECL through the so-called co-reactant pathway. Figure 8a-c show the reduction waves corresponding to the reduction of BPO (red traces) and the corresponding photocurrent generated (blue traces). ECL intensity maxima of 3000, 1280 and $80 \mathrm{nA}$ were measured for PPOCzPN, PPSCzPN and DiPPOCzPN, respectively. PPOCzPN showed the strongest ECL response, which was almost three-fold stronger than that of PPSCzPN and 38 times that of DiPPOCzPN. From previous theoretical and experimental studies, ${ }^{90}$ benzoate radicals have a redox potential of $1.5 \mathrm{~V}$, which is sufficient to oxidize the three TADF compounds in this study, and for this co-reactant ECL pathway to provide more energy than the annihilation ECL pathway. The difference in 
emission intensities compared to the annihilation pathway is likely due to this available energy increase.

The BPO co-reactant ECL efficiencies for PPOCzPN, PPSCzPN and DiPPOCzPN were determined to be 17, 6 and 1\%, respectively, using Eq. 2 (Table 6). Interestingly, this ECL efficiency difference between PPOCzPN and DiPPOCzPN resembles the difference in $\Phi_{\mathrm{PL}}$ for the emitters in a PMMA film (56 to $28 \%$ ) more than it does the $\Phi_{\mathrm{PL}}$ difference in DCM (51 to $61 \%$ ). This indicates that the ECL co-reactant pathway for DiPPOCzPN has an additional nonradiative decay pathway. In ECL co-reactant systems, the lifetime of the radical poses less of an issue than in annihilation experiments, since radical reactants are generated almost simultaneously, typically allowing ECL enhancement in solution. Now that radical stability is less of a factor, $\mathbf{P P O C z P N}^{\bullet}$ appears to have a higher reactivity than DiPPOCzPN $\mathbf{~}^{\bullet-}$ and $\mathbf{P P S C z P N}{ }^{\bullet-}$ towards BPO. A higher anion reactivity for PPOCzPN was noted during pulsing experiments (Figure 2d-f) and appears to have been observed again in the BPO experiments. With increased available energy, it appears that PPOCzPN is the strongest emitter and maintains its position as the best candidate for optoelectronics.

\subsection{ECL Spectroscopy}

Figures 8d-f show spooling ECL spectroscopy performed on all co-reactant systems described herein. The ECL spooling spectra add a new data dimension of wavelength to the same spectra seen in Figures 8a-c. The BPO co-reactant systems show a light green emission for all species with maximum emission CIE coordinates of $(0.29,0.40),(0.31,0.42)$ and $(0.33,0.38)$ for PPOCzPN, PPSCzPN and DiPPOCzPN, respectively. In contrast to the annihilation pathway and also observed in the PL study, the DiPPOCzPN/BPO co-reactant system has the most redshifted emission spectrum (Table 4 and Figure 8f), rather than the most blue-shifted. This large red-shift and relative drop in efficiency indicate that DiPPOCzPN may suffer from exciplex formation under these conditions. Exciplexes are heterodimeric species created by intermolecular interactions between a hole on the donor moiety of one molecule and the electron on the acceptor moiety of a second molecule. ${ }^{110}$ Exciplexes typically have smaller HOMO-LUMO gaps than either of the constituent components, thereby resulting in an observed red-shifted emission. Exciplex formation in OLEDs with luminophores substituted by diphenyl phosphine oxide substituents have been reported by Tourneur et al., who harnessed this exciplex formation to produce emissions from both intramolecular and intermolecular charge-transfer states yielding white light OLEDs. ${ }^{111}$ The exciplex identified during DiPPOCzPN/BPO co-reactant analysis appears not to be affected by an increasing concentration of reduced species in the vicinity of the electrode, since as the cyclic voltammogram progresses, no shift in emission maxima is seen (insets of Figure 8d-f with expansions of the insets in Figure S48). Additionally, there was no identified exciplex formation during the annihilation studies with DiPPOCzPN. The most probable explanation for the observed red-shifted emission is due to the exciplex between DiPPOCzPN'- and $\mathrm{Bz}^{\bullet+}$. Newcomb et al. has identified the necessity to screen potential TADF OLED emitters for exciplex formation, as the presence of exciplexes was shown to lower device lifetime. ${ }^{12}$ Masui et al. also identified large EQE roll-off in OLEDs caused by charge imbalance in the emissive layer that are exacerbated at higher current densities. ${ }^{101,113}$ The exciplex formation in these compounds is characteristic of compounds that also exhibit OLPL, thereby supporting OLECL emission as the dominant mechanism. Interestingly, the longest pulsing ECL onset delay belonged to DiPPOCzPN, which was the only compound to form a very evident exciplex during BPO co-reactant analysis. $\mathbf{2 C z P N}$ showed emissions centered at $550 \mathrm{~nm}$, resembling the emissions seen in the DiPPOCzPN/BPO 
coreactant studies in Figure S38c-d. This center wavelength is also $50 \mathrm{~nm}$ red-shifted from the 2CzPN PL studies likely due to a similar exciplex formation.

In a similar manner to the BPO co-reactant, $5 \mathrm{mM}$ tripropylamine (TPrA) was added as an alternative co-reactant (Figure S49-S50). A thorough discussion of the TPrA co-reactant systems follows Figures S49 in the supporting information. Under these conditions, the PPSCzPN/TPrA system behaved in the exact same manner as the DiPPOCzPN/BPO system, likely indicating a similar exciplex formation. There were no excimers identified in the PPSCzPN annihilation mechanism, indicating that the interactions between $\mathbf{P P S C z P N}^{\cdot+}$ and $\mathbf{N P r}_{3}{ }^{\bullet}$ may be responsible for the exciplex formation. The PPSCzPN/TPrA system also seems to be concentration-dependent where an increasing overpotential that creates more PPSCzPN ${ }^{\bullet+}$ in the vicinity of the electrode gradually causes a red-shift. The compounds with the longest onset to ECL emission, longest time to achieve a maximum ECL intensity, longest ECL decay time, largest $\triangle \mathrm{E}_{\mathrm{ST}}$ and weakest ECL efficiencies likely form exciplexes under co-reactant conditions. These correlations and relationships all support the putative OLECL emission mechanism. 

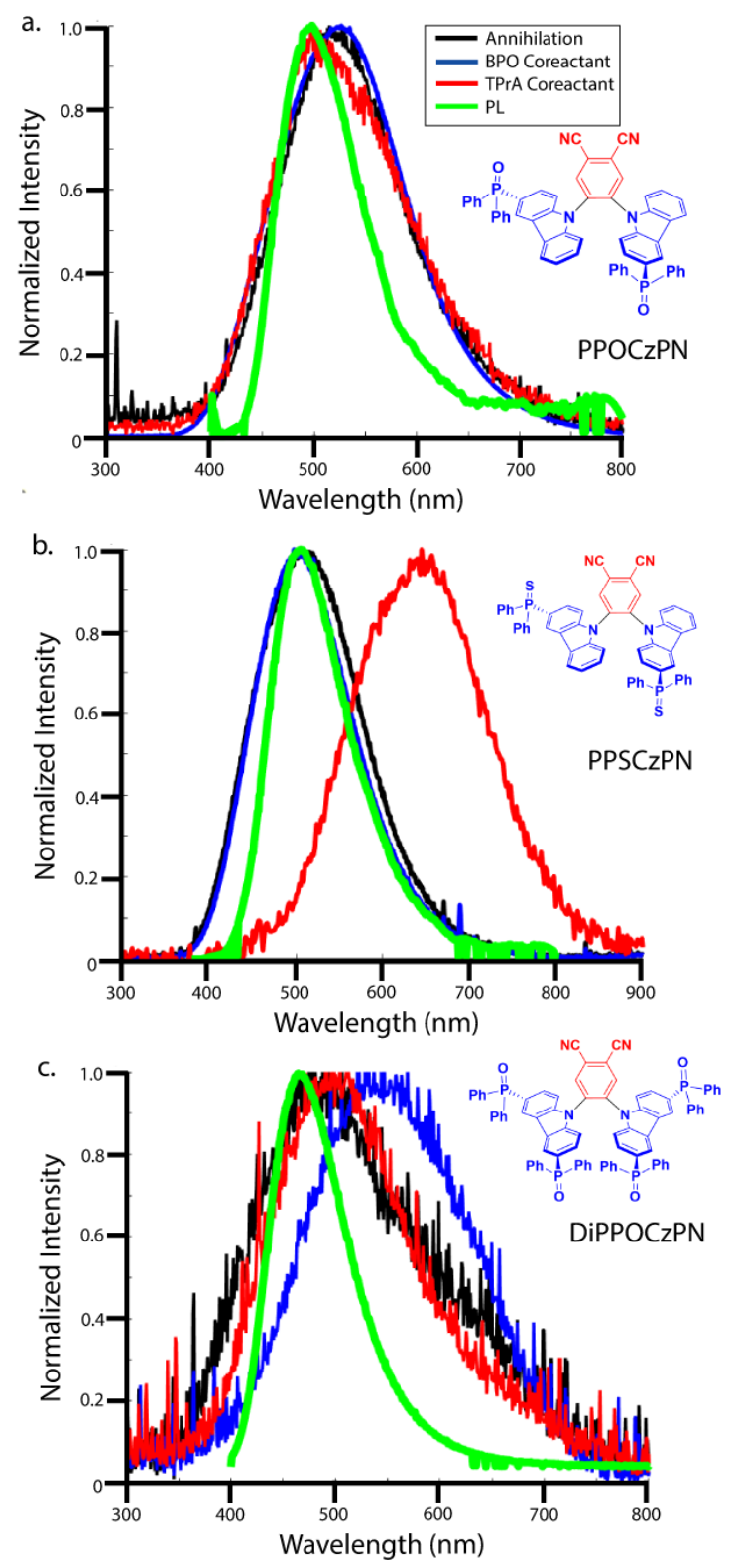

Figure 9. Accumulated ECL spectra during different light generation methods. The annihilation ECL, BPO co-reactant ECL, TPrA co-reactant ECL and photoluminescence emissions are coloured in black, blue, red and green traces for (a) PPOCzPN, (b) PPSCzPN and (c) DiPPOCzPN, respectively.

In Figure 9, the normalized accumulation spectra of all ECL processes discussed so far are overlapped, with ECL peak wavelengths summarized in Table 6. A slight red-shift is seen for all ECL emissions relative to the PL emissions, which is likely due to the more concentrated solutions used in the ECL measurements that result in increased self-absorption of the higher energy components of the emission spectra in the ECL studies. The PL and ECL spectra for PPOCzPN do not shift, indicating a common excited state accessed in all processes. However, there is a large 
red-shift observed for both the DiPPOCzPN/BPO co-reactant pathway and for the PPSCzPN/TPrA co-reactant pathway. These red-shifts in the ECL spectra typically indicate exciplex formation.

\section{Conclusions}

Herein, we detailed the synthesis and characterization of three new $2 \mathbf{C z P N}$ derivatives with phosphine chalcogenide oxide groups substituted on the donor moiety. This strategy successfully stabilized the HOMO level of the donor, confirmed by cyclic voltammetry. By extension, the lowest-lying charge-transfer state was shifted to higher energy, thereby promoting a deeper blue emission, confirmed by photoluminescence studies. Surprisingly, the same trends are not recovered when measuring emissions in apolar media, thus highlighting solvatochromic effects on the TADF properties of the $\mathbf{2 C z P N}$ derivatives. Quantum-chemical calculations support these solvatochromic observations. Electrochemiluminescence studies in the desirable polar medium revealed a heretofore undocumented emission mechanism under electrical excitation in solution. Delayed ECL onset times relative to common ECL emitters in annihilation pathway revealed that organic long-persistent emission was operational and that the delay in the onset times correlated with the $\Delta E_{\mathrm{ST}}$ of the emitters. PPOCzPN, which has the smallest $\Delta E_{\mathrm{ST}}$, yielded the strongest, fastest, and most efficient ECL emissions when using BPO co-reactant. Thus, this compound should be the most suitable for optoelectronic applications after considering radical stability, emission stability, emission efficiency and emission intensity. Studies on ECL spooling spectroscopy and accumulated ECL spectroscopy identified that an exciplex of DiPPOCzPN forms while in the presence of radical anions. This work demonstrates how ECL can be used to guide and screen TADF compound suitability for optoelectronic applications such as OLEDs.

\section{Supporting Information}

Experimental section, general synthetic procedure, photophysical measurements, electrochemical measurements, theoretical modelling, synthesis, chemical characterization, X-ray crystallography, additional electrochemical experiments and electrochemiluminescence.

\section{Acknowledgments}

The authors thank Dr Sai Kiran Rajendran for providing PL and TCSPC data of $2 \mathbf{C z P N}$ in neat film. SK acknowledges the financial support from European Union's Horizon 2020 research and innovation programme under Marie Skłodowska Curie Individual Fellowship (MCIF; Agreement No. 748430-THF-OLED). P. R acknowledges support from a Marie Skłodowska-Curie Individual Fellowship (No. 749557). We thank the EPSRC UK National Mass Spectrometry Facility at Swansea University for analytical services. The work has been supported in Mons by European Union through the Interreg V initiative France-Wallonie-Vlaanderen project LUMINOPTEX and the Belgian National Fund for Scientific Research (FRS-FNRS). Computational resources were provided by the Consortium des Équipements de Calcul Intensif (CÉCI) funded by F.R.S.-FNRS under Grant 2.5020.11. J.C. is an FNRS research director. Y.O. acknowledges funding by the Fonds de la Recherche Scientifique-FNRS under Grant n ${ }^{\circ}$ F.4534.21 (MIS-IMAGINE). We acknowledge the research support from Natural Sciences and Engineering Research Council Canada (NSERC, DG RGPIN-2013-201697, DG RGPIN-2018-06556, and SPG STPGP-2016- 
493924), Canada Foundation of Innovation, Ontario Innovation Trust (CFI/OIT, 9040) and Western University. J.R.A. appreciates the Ontario graduate scholarships (2018-2021).

\section{Author Contributions}

S.K., M.Y.W., and E.Z.-C. designed and synthesized the materials. S.K., P.R. and P.T. carried out the photophysical study. J.R.A and Z.D. performed the electrochemical and ECL studies. D.B.C. and A.M.Z.S. solved the structure of the single crystals. P.T., Y.O., J.C., R.L., and P.V. carried out the theoretical modeling. S.K., E.Z.-C., J.R.A., Z.D., P.T., Y.O., J.C. wrote the manuscript. Y.O., J.C., Z.D. and E.Z.-C. supervised the project.

\section{Corresponding Authors}

Yoann Olivier - Unité de Chimie Physique Théorique et Structurale (UCPTS) \& Laboratoire de Physique du Solide (LPS), Namur Institute of Structured Matter (NISM), University of Namur, Namur, Belgium; ORCID: https://orcid.org/0000-0003-2193-1536; $\quad$ E-mail: yoann.olivier@unamur.be

Jerome Cornil - Laboratory for Chemistry of Novels Materials, University of Mons, Mons, Belgium; ORCID: E-mail: Jerome.Cornil@umons.ac.be

Zhifeng Ding - Department of Chemistry, The University of Western Ontario, London, Ontario N6A 3K7, Canada; E-mail: zfding@uwo.ca

Eli Zyman-Colman - Organic Semiconductor Centre, EaStCHEM School of Chemistry, University of St Andrews, St Andrews, Fife, UK, KY16 9ST; ORCID: http://orcid.org/0000-00017183-6022; E-mail: eli.zysman-colman@st-andrews.ac.uk

\section{References}

1. Sun, Y.; Giebink, N. C.; Kanno, H.; Ma, B.; Thompson, M. E.; Forrest, S. R., Management of Singlet and Triplet Excitons for Efficient White Organic Light-Emitting Devices. Nature 2006, 440 (7086), 908-12.

2. Adachi, C.; Baldo, M. A.; Thompson, M. E.; Forrest, S. R., Nearly $100 \%$ internal phosphorescence efficiency in an organic light-emitting device. J. Appl. Phys. 2001, 90 (10), 5048-5051.

3. Klubek, K. P.; Tang, C. W.; Rothberg, L. J., Investigation of Blue Phosphorescent Organic LightEmitting Diode Host and Dopant Stability. Org. Electron. 2014, 15 (7), 1312-1316.

4. Huang, T.; Jiang, W.; Duan, L., Recent Progress in Solution Processable TADF Materials for Organic Light-Emitting Diodes. J. Mater. Chem. C 2018, 6 (21), 5577-5596.

5. $\quad$ Chen, X.-K.; Kim, D.; Brédas, J.-L., Thermally Activated Delayed Fluorescence (TADF) Path Toward Efficient Electroluminescence in Purely Organic Materials: Molecular Level Insight. Acc. Chem. Res. 2018, 51 (9), 2215-2224.

6. Jeon, S. K.; Lee, H. L.; Yook, K. S.; Lee, J. Y., Recent Progress of the Lifetime of Organic LightEmitting Diodes Based on Thermally Activated Delayed Fluorescent Material. Adv. Mater. 2019, 31 (34), 1803524.

7. Godumala, M.; Choi, S.; Cho, M. J.; Choi, D. H., Recent Breakthroughs in Thermally Activated Delayed Fluorescence Organic Light Emitting Diodes Containing Non-Doped Emitting Layers. J. Mater. Chem. C 2019, 7 (8), 2172-2198.

8. Data, P.; Takeda, Y., Recent Advancements in and the Future of Organic Emitters: TADF- and RTP-Active Multifunctional Organic Materials. Chem. Asian J. 2019, 14 (10), 1613-1636.

9. Olivier, Y.; Sancho-Garcia, J. C.; Muccioli, L.; D’Avino, G.; Beljonne, D., Computational Design of Thermally Activated Delayed Fluorescence Materials: The Challenges Ahead. J. Phys. Chem. Lett. 2018, $9(20), 6149-6163$. 
10. Uoyama, H.; Goushi, K.; Shizu, K.; Nomura, H.; Adachi, C., Highly Efficient Organic Light Emitting Diodes from Delayed Fluorescence. Nature 2012, 492 (7428), 234-238.

11. de Silva, P.; Kim, C. A.; Zhu, T.; Van Voorhis, T., Extracting Design Principles for Efficient Thermally Activated Delayed Fluorescence (TADF) from a Simple Four-State Model. Chem. Mater. 2019, 31 (17), 6995-7006.

12. Nobuyasu, R. S.; Ren, Z.; Griffiths, G. C.; Batsanov, A. S.; Data, P.; Yan, S.; Monkman, A. P.; Bryce, M. R.; Dias, F. B., Rational Design of TADF Polymers Using a Donor-Acceptor Monomer with Enhanced TADF Efficiency Induced by the Energy Alignment of Charge Transfer and Local Triplet Excited States. Adv. Opt. Mater. 2016, 4 (4), 597-607.

13. Reineke, S.; Lindner, F.; Schwartz, G.; Seidler, N.; Walzer, K.; Lüssem, B.; Leo, K., White Organic Light-Emitting Diodes With Fluorescent Tube Efficiency. Nature 2009, 459 (7244), 234-238.

14. Tao, Y.; Yuan, K.; Chen, T.; Xu, P.; Li, H.; Chen, R.; Zheng, C.; Zhang, L.; Huang, W., Thermally Activated Delayed Fluorescence Materials Towards the Breakthrough of Organoelectronics. Adv. Mater. 2014, 26 (47), 7931-7958.

15. Spuling, E.; Sharma, N.; Samuel, I. D. W.; Zysman-Colman, E.; Bräse, S., (Deep) Blue ThroughSpace Conjugated TADF Emitters Based on [2.2]Paracyclophanes. Chem. Commun. 2018, 54 (67), 92789281.

16. Hatakeyama, T.; Shiren, K.; Nakajima, K.; Nomura, S.; Nakatsuka, S.; Kinoshita, K.; Ni, J.; Ono, Y.; Ikuta, T., Ultrapure Blue Thermally Activated Delayed Fluorescence Molecules: Efficient HOMOLUMO Separation by the Multiple Resonance Effect. Adv. Mater. 2016, 28 (14), 2777-2781.

17. Auffray, M.; Kim, D. H.; Kim, J. U.; Bencheikh, F.; Kreher, D.; Zhang, Q.; D'Aléo, A.; Ribierre, J.-C.; Mathevet, F.; Adachi, C., Dithia[3.3]paracyclophane Core: A Versatile Platform for Triplet State Fine-Tuning and Through-Space TADF Emission. Chem. Asian J. 2019, 14 (11), 1921-1925.

18. Lee, J.; Aizawa, N.; Yasuda, T., Molecular Engineering of Phosphacycle-Based Thermally Activated Delayed Fluorescence Materials for Deep-Blue OLEDs. J. Mater. Chem. C 2018, 6 (14), 35783583.

19. Liang, X.; Yan, Z.-P.; Han, H.-B.; Wu, Z.-G.; Zheng, Y.-X.; Meng, H.; Zuo, J.-L.; Huang, W., Peripheral Amplification of Multi-Resonance Induced Thermally Activated Delayed Fluorescence for Highly Efficient OLEDs. Angew. Chem. Int. Ed. 2018, 57 (35), 11316-11320.

20. Cho, Y. J.; Jeon, S. K.; Lee, S.-S.; Yu, E.; Lee, J. Y., Donor Interlocked Molecular Design for Fluorescence-like Narrow Emission in Deep Blue Thermally Activated Delayed Fluorescent Emitters. Chem. Mater. 2016, 28 (15), 5400-5405.

21. Mei, L.; Hu, J.; Cao, X.; Wang, F.; Zheng, C.; Tao, Y.; Zhang, X.; Huang, W., The InductiveEffect of Electron Withdrawing Trifluoromethyl for Thermally Activated Delayed Fluorescence: Tunable Emission from Tetra- to Penta-Carbazole in Solution Processed Blue OLEDs. Chem. Commun. 2015, 51 (65), 13024-13027.

22. Wang, H.; Xie, L.; Peng, Q.; Meng, L.; Wang, Y.; Yi, Y.; Wang, P., Novel Thermally Activated Delayed Fluorescence Materials-Thioxanthone Derivatives and Their Applications for Highly Efficient OLEDs. Adv. Mater. 2014, 26 (30), 5198-5204.

23. Furue, R.; Nishimoto, T.; Park, I. S.; Lee, J.; Yasuda, T., Aggregation-Induced Delayed Fluorescence Based on Donor/Acceptor-Tethered Janus Carborane Triads: Unique Photophysical Properties of Nondoped OLEDs. Angew. Chem. Int. Ed. 2016, 55 (25), 7171-7175.

24. Higginbotham, H. F.; Pander, P.; Rybakiewicz, R.; Etherington, M. K.; Maniam, S.; Zagorska, M.; Pron, A.; Monkman, A. P.; Data, P., Triphenylamine Disubstituted Naphthalene Diimide: Elucidation of Excited States Involved in TADF and Application in Near-Infrared Organic Light Emitting Diodes. $J$. Mater. Chem. C 2018, 6 (30), 8219-8225.

25. Xie, G.; Li, X.; Chen, D.; Wang, Z.; Cai, X.; Chen, D.; Li, Y.; Liu, K.; Cao, Y.; Su, S.-J., Evaporation- and Solution-Process-Feasible Highly Efficient Thianthrene-9,9',10,10'-Tetraoxide-Based Thermally Activated Delayed Fluorescence Emitters with Reduced Efficiency Roll-Off. Adv. Mater. 2016, 28 (1), 181-187. 
26. Lin, T.-A.; Chatterjee, T.; Tsai, W.-L.; Lee, W.-K.; Wu, M.-J.; Jiao, M.; Pan, K.-C.; Yi, C.-L.; Chung, C.-L.; Wong, K.-T.; Wu, C.-C., Sky-Blue Organic Light Emitting Diode with 37\% External Quantum Efficiency Using Thermally Activated Delayed Fluorescence from Spiroacridine-Triazine Hybrid. Adv. Mater. 2016, 28 (32), 6976-6983.

27. Takahashi, T.; Shizu, K.; Yasuda, T.; Togashi, K.; Adachi, C., Donor-Acceptor-Structured 1,4Diazatriphenylene Derivatives Exhibiting Thermally Activated Delayed Fluorescence: Design and Synthesis, Photophysical Properties and OLED Characteristics. Sci. Technol. Adv. Mat. 2014, 15 (3), 034202.

28. Tanaka, H.; Shizu, K.; Nakanotani, H.; Adachi, C., Twisted Intramolecular Charge Transfer State for Long-Wavelength Thermally Activated Delayed Fluorescence. Chem. Mater. 2013, 25 (18), 37663771.

29. Lee, J.; Shizu, K.; Tanaka, H.; Nomura, H.; Yasuda, T.; Adachi, C., Oxadiazole- and TriazoleBased Highly-Efficient Thermally Activated Delayed Fluorescence Emitters for Organic Light-Emitting Diodes. J. Mater. Chem. C 2013, 1 (30), 4599-4604.

30. Duan, C.; Li, J.; Han, C.; Ding, D.; Yang, H.; Wei, Y.; Xu, H., Multi-dipolar Chromophores Featuring Phosphine Oxide as Joint Acceptor: A New Strategy toward High-Efficiency Blue Thermally Activated Delayed Fluorescence Dyes. Chem. Mater. 2016, 28 (16), 5667-5679.

31. Chen, D.-Y.; Liu, W.; Zheng, C.-J.; Wang, K.; Li, F.; Tao, S. L.; Ou, X.-M.; Zhang, X.-H., Isomeric Thermally Activated Delayed Fluorescence Emitters for Color Purity-Improved Emission in Organic Light-Emitting Devices. ACS Appl. Mater. Inter. 2016, 8 (26), 16791-16798.

32. Aizawa, N.; Tsou, C.-J.; Park, I. S.; Yasuda, T., Aggregation-Induced Delayed Fluorescence From Phenothiazine-Containing Donor-Acceptor Molecules for High-Efficiency Non-Doped Organic Light-Emitting Diodes. Polym. J. 2017, 49 (1), 197-202.

33. Xu, S.; Liu, T.; Mu, Y.; Wang, Y.-F.; Chi, Z.; Lo, C.-C.; Liu, S.; Zhang, Y.; Lien, A.; Xu, J., An Organic Molecule with Asymmetric Structure Exhibiting Aggregation-Induced Emission, Delayed Fluorescence, and Mechanoluminescence. Angew. Chem. Int. Ed. 2015, 54 (3), 874-878.

34. Lee, D. R.; Kim, B. S.; Lee, C. W.; Im, Y.; Yook, K. S.; Hwang, S.-H.; Lee, J. Y., Above 30\% External Quantum Efficiency in Green Delayed Fluorescent Organic Light-Emitting Diodes. ACS Appl. Mater. Inter. 2015, 7 (18), 9625-9629.

35. Jeon, S. K.; Lee, J. Y., Highly Efficient Exciplex Organic Light-Emitting Diodes by Exciplex Dispersion in the Thermally Activated Delayed Fluorescence Host. Org. Electron. 2020, 76, 105477.

36. Wex, B.; Kaafarani, B. R., Perspective on Carbazole-Based Organic Compounds as Emitters and Hosts in TADF Applications. J. Mater. Chem. C 2017, 5 (34), 8622-8653.

37. Cho, Y. J.; Yook, K. S.; Lee, J. Y., High Efficiency in a Solution-Processed Thermally Activated Delayed-Fluorescence Device Using a Delayed-Fluorescence Emitting Material with Improved Solubility. Adv. Mater. 2014, 26 (38), 6642-6646.

38. Wu, S.; Aonuma, M.; Zhang, Q.; Huang, S.; Nakagawa, T.; Kuwabara, K.; Adachi, C., HighEfficiency Deep-Blue Organic Light-Emitting Diodes Based on a Thermally Activated Delayed Fluorescence Emitter. J. Mater. Chem. C 2014, 2 (3), 421-424.

39. Kim, G. H.; Lampande, R.; Park, M. J.; Bae, H. W.; Kong, J. H.; Kwon, J. H.; Park, J. H.; Park, Y. W.; Song, C. E., Highly Efficient Bipolar Host Materials with Indenocarbazole and Pyrimidine Moieties for Phosphorescent Green Light-Emitting Diodes. J. Phys. Chem. C 2014, 118 (49), 2875728763.

40. Lee, D. R.; Choi, J. M.; Lee, C. W.; Lee, J. Y., Ideal Molecular Design of Blue Thermally Activated Delayed Fluorescent Emitter for High Efficiency, Small Singlet-Triplet Energy Splitting, Low Efficiency Roll-Off, and Long Lifetime. ACS Appl. Mater. Inter. 2016, 8 (35), 23190-23196.

41. Sun, K.; Sun, Y.; Huang, T.; Luo, J.; Jiang, W.; Sun, Y., Design Strategy of Yellow Thermally Activated Delayed Fluorescent Dendrimers and Their Highly Efficient Non-Doped Solution-Processed OLEDs with Low Driving Voltage. Org. Electron. 2017, 42, 123-130. 
42. Albrecht, K.; Matsuoka, K.; Fujita, K.; Yamamoto, K., Carbazole Dendrimers as SolutionProcessable Thermally Activated Delayed-Fluorescence Materials. Angew. Chem. Int. Ed. 2015, 54 (19), $5677-5682$.

43. Lee, D. R.; Hwang, S.-H.; Jeon, S. K.; Lee, C. W.; Lee, J. Y., Benzofurocarbazole and Benzothienocarbazole as Donors for Improved Quantum Efficiency in Blue Thermally Activated Delayed Fluorescent Devices. Chem. Commun. 2015, 51 (38), 8105-8107.

44. Sharma, N.; Spuling, E.; Mattern, Cornelia M.; Li, W.; Fuhr, O.; Tsuchiya, Y.; Adachi, C.; Bräse, S.; Samuel, I. D. W.; Zysman-Colman, E., Turn on of Sky-Blue Thermally Activated Delayed Fluorescence and Circularly Polarized Luminescence (CPL) via Increased Torsion by a Bulky Carbazolophane Donor. Chem. Sci. 2019, 10 (27), 6689-6696.

45. Huang, B.; Jiang, W.; Liu, Y. Y.; Zhang, Y. A.; Yang, Y. P.; Dai, Y.; Ban, X. X.; Xu, H. G.; Sun, Y. M., Thermally Activated Delayed Fluorescence Materials Based on Carbazole/Sulfone. Adv. Mater. Res. 2014, 1044-1045, 158-163.

46. Im, Y.; Lee, J. Y., Effect of the Position of Nitrogen in Pyridoindole on Photophysical Properties and Device Performances of $\alpha$-, $\beta$-, $\gamma$-Carboline Based High Triplet Energy Host Materials for deep Blue Devices. Chem. Commun. 2013, 49 (53), 5948-5950.

47. Jun, J.-W.; Lee, K.-M.; Kim, O. Y.; Lee, J. Y.; Hwang, S.-H., Synthesis of a

Dibenzothiophene/Carboline/Carbazole Hybrid Bipolar Host Material for Green Phosphorescent OLEDs. Synth. Met. 2016, 213, 7-11.

48. Byeon, S. Y.; Jeon, S. K.; Hwang, S.-H.; Lee, J. Y., Carbazole-Carboline Core as a Backbone Structure of High Triplet Energy Host Materials. Dyes Pigments 2015, 120, 258-264.

49. Zhang, Z.; Xie, J.; Wang, Z.; Shen, B.; Wang, H.; Li, M.; Zhang, J.; Cao, J., Manipulation of Electron Deficiency of $\delta$-Carboline Derivatives as Bipolar Hosts for Blue Phosphorescent Organic LightEmitting Diodes with High Efficiency at $1000 \mathrm{~cd} \mathrm{~m}^{-2}$. J. Mater. Chem. C 2016, 4 (19), 4226-4235.

50. Lee, C. W.; Lee, J. Y., Above 30\% External Quantum Efficiency in Blue Phosphorescent Organic Light-Emitting Diodes Using Pyrido[2,3-b]indole Derivatives as Host Materials. Adv. Mater. 2013, 25 (38), 5450-5454.

51. Lai, C.-C.; Huang, M.-J.; Chou, H.-H.; Liao, C.-Y.; Rajamalli, P.; Cheng, C.-H., mIndolocarbazole Derivative as a Universal Host Material for RGB and White Phosphorescent OLEDs. Adv. Funct. Mater. 2015, 25 (34), 5548-5556.

52. Yook, K. S.; Lee, J. Y., Bipolar Host Materials for Organic Light-Emitting Diodes. Chem. Rec. 2016, $16(1), 159-172$.

53. Lin, M.-S.; Chi, L.-C.; Chang, H.-W.; Huang, Y.-H.; Tien, K.-C.; Chen, C.-C.; Chang, C.-H.; Wu, C.-C.; Chaskar, A.; Chou, S.-H.; Ting, H.-C.; Wong, K.-T.; Liu, Y.-H.; Chi, Y., A DiarylboraneSubstituted Carbazole as a Universal Bipolar Host Material for Highly Efficient Electrophosphorescence Devices. J. Mater. Chem. 2012, 22 (3), 870-876.

54. $\quad$ Lin, M.-S.; Yang, S.-J.; Chang, H.-W.; Huang, Y.-H.; Tsai, Y.-T.; Wu, C.-C.; Chou, S.-H.; Mondal, E.; Wong, K.-T., Incorporation of a CN Group into mCP: A New Bipolar Host Material for Highly Efficient Blue and White Electrophosphorescent Devices. J. Mater. Chem. 2012, 22 (31), 1611416120.

55. Oh, C. S.; Choi, J. M.; Lee, J. Y., Chemical Bond Stabilization and Exciton Management by CN Modified Host Material for Improved Efficiency and Lifetime in Blue Phosphorescent Organic LightEmitting Diodes. Adv. Opt. Mater. 2016, 4 (8), 1281-1287.

56. Zhang, Q.; Li, J.; Shizu, K.; Huang, S.; Hirata, S.; Miyazaki, H.; Adachi, C., Design of Efficient Thermally Activated Delayed Fluorescence Materials for Pure Blue Organic Light Emitting Diodes. $J$. Am. Chem. Soc. 2012, 134 (36), 14706-14709.

57. Hirata, S.; Sakai, Y.; Masui, K.; Tanaka, H.; Lee, S. Y.; Nomura, H.; Nakamura, N.; Yasumatsu, M.; Nakanotani, H.; Zhang, Q.; Shizu, K.; Miyazaki, H.; Adachi, C., Highly Efficient Blue Electroluminescence Based on Thermally Activated Delayed Fluorescence. Nat. Mater. 2015, 14 (3), 330-336. 
58. Colella, M.; Danos, A.; Monkman, A. P., Less Is More: Dilution Enhances Optical and Electrical Performance of a TADF Exciplex. J. Phys. Chem. Lett. 2019, 10 (4), 793-798.

59. Masui, K.; Nakanotani, H.; Adachi, C., Analysis of Exciton Annihilation in High-Efficiency SkyBlue Organic Light-Emitting Diodes With Thermally Activated Delayed Fluorescence. Org. Electron. 2013, 14 (11), 2721-2726.

60. Hehre, W. J.; Ditchfield, R.; Pople, J. A., Self-Consistent Molecular Orbital Methods. XII. Further Extensions of Gaussian-Type Basis Sets for Use in Molecular Orbital Studies of Organic Molecules. J. Chem. Phys. 1972, 56 (5), 2257-2261.

61. Adsetts, J. R.; Zhang, R.; Yang, L.; Chu, K.; Wong, J. M.; Love, D. A.; Ding, Z., Efficient White Electrochemiluminescent Emission From Carbon Quantum Dot Films. Front. Chem. 2020, 8, 580022.

62. Chu, K.; Adsetts, J. R.; He, S.; Zhan, Z.; Yang, L.; Wong, J. M.; Love, D. A.; Ding, Z.,

Electrogenerated Chemiluminescence and Electroluminescence of N-Doped Graphene Quantum Dots

Fabricated from an Electrochemical Exfoliation Process in Nitrogen-Containing Electrolytes. Chem. Eur. J. 2020, 26 (68), 15892-15900.

63. Adsetts, J. R.; Hoesterey, S.; Gao, C.; Love, D. A.; Ding, Z., Electrochemiluminescence and Photoluminescence of Carbon Quantum Dots Controlled by Aggregation-Induced Emission, Aggregation-Caused Quenching and Interfacial Reactions. Langmuir 2020, 36 (47), 14432-14442. 64. Chu, K.; Adsetts, J. R.; Moore, C.; Ding, Z., Spooling Electroluminescence Spectroscopy of $\mathrm{Ru}(\mathrm{bpy})_{3}{ }^{2+}$ Light-Emitting Electrochemical Cells with an Atomic Layer Deposited Zinc Oxide ElectronTransporting/Hole-Blocking Interlayer. ACS Appl. Eletron. Mater. 2020, 2 (12), 3825-3830.

65. Adsetts, J. R.; Ding, Z., Film Electrochemiluminescence Controlled by Interfacial Reactions Along with Aggregation-, Matrix-Coordination-, and Crystallization-Induced Emissions. Chempluschem 2021, 86 (1), 155-165.

66. Yang, L.; Koo, D.; Wu, J.; Wong, J. M.; Day, T.; Zhang, R.; Kolongoda, H.; Liu, K.; Wang, J.; Ding, Z.; Pagenkopf, B. L., Benzosiloles with Crystallization-Induced Emission Enhancement of Electrochemiluminescence: Synthesis, Electrochemistry, and Crystallography. Chemistry 2020, 26 (51), 11715-11721.

67. Wong, J. M.; Zhang, R.; Xie, P.; Yang, L.; Zhang, M.; Zhou, R.; Wang, R.; Shen, Y.; Yang, B.; Wang, H. B.; Ding, Z., Revealing Crystallization-Induced Blue-Shift Emission of a Di-Boron Complex by Enhanced Photoluminescence and Electrochemiluminescence. Angew. Chem. Int. Ed. Engl. 2020, 59 (40), 17461-17466.

68. Ege, D.; Becker, W. G.; Bard, A. J., Electrogenerated Chemiluminescent Determination of $\mathrm{Ru}(\mathrm{bpy}){ }_{3}{ }^{2+}$ at Low Levels. Anal. Chem. 1984, 56, 2413-2417.

69. Chandross, E. A.; Sonntag, F. I., A Novel Chemiluminescent Electron-Transfer Reaction. J. Am. Chem. Soc. 1964, 86 (15), 3179-3180.

70. Hercules, D. M., Chemiluminescence Resulting From Electrochemically Generated Species. Science 1964, 145 (3634), 808-809.

71. Santhanam, K. S. V.; Bard, A. J., Chemiluminescence of Electrogenerated 9,10Diphenylathracene Anion Radical. J. Am. Chem. Soc. 1965, 87 (1), 139-140.

72. Liu, Z.; Qi, W.; Xu, G., Recent Advances in Electrochemiluminescence. Chem. Soc. Rev. 2015, 44 (10), 3117-42.

73. Ishimatsu, R.; Matsunami, S.; Kasahara, T.; Mizuno, J.; Edura, T.; Adachi, C.; Nakano, K.; Imato, T., Electrogenerated Chemiluminescence of Donor-Acceptor Molecules with Thermally Activated Delayed Fluorescence. Angew. Chem. Int. Ed. 2014, 53 (27), 6993-6996.

74. Etherington, M. K.; Gibson, J.; Higginbotham, H. F.; Penfold, T. J.; Monkman, A. P., Revealing the Spin-Vibronic Coupling Mechanism of Thermally Activated Delayed Fluorescence. Nat. Commun. 2016, 7, 13680.

75. Zeng, Z.; Huang, P.; Kong, Y.; Tong, L.; Zhang, B.; Luo, Y.; Chen, L.; Zhang, Y.; Han, D.; Niu, L., Nanoencapsulation Strategy: Enabling Electrochemiluminescence of Thermally Activated Delayed Fluorescence (TADF) Emitters in Aqueous Media. Chem. Commun. 2021. 
76. Ishimatsu, R.; Edura, T.; Adachi, C.; Nakano, K.; Imato, T., Photophysical Properties and Efficient, Stable, Electrogenerated Chemiluminescence of Donor-Acceptor Molecules Exhibiting Thermal Spin Upconversion. Chem. Eur. J. 2016, 22 (14), 4889-4898.

77. Kapturkiewicz, A., Electrochemical Generation of Excited Intramolecular Charge-Transfer States. ChemElectroChem 2017, 4 (7), 1604-1638.

78. $\quad$ Wong, M. Y.; Hedley, G. J.; Xie, G.; Kölln, L. S.; Samuel, I. D. W.; Pertegás, A.; Bolink, H. J.; Zysman-Colman, E., Light-Emitting Electrochemical Cells and Solution-Processed Organic LightEmitting Diodes Using Small Molecule Organic Thermally Activated Delayed Fluorescence Emitters. Chem. Mater. 2015, 27 (19), 6535-6542.

79. Wong, M. Y.; Krotkus, S.; Copley, G.; Li, W.; Murawski, C.; Hall, D.; Hedley, G. J.; Jaricot, M.; Cordes, D. B.; Slawin, A. M. Z.; Olivier, Y.; Beljonne, D.; Muccioli, L.; Moral, M.; Sancho-Garcia, J.-C.; Gather, M. C.; Samuel, I. D. W.; Zysman-Colman, E., Deep-Blue Oxadiazole-Containing Thermally Activated Delayed Fluorescence Emitters for Organic Light-Emitting Diodes. ACS Appl. Mater. Inter. 2018, 10 (39), 33360-33372.

80. Melhuish, W. H., Quantum Efficiencies of Fluorescence of Organic Substances: Effect of Solvent and Concentration of the Fluorescent Solute. J. Phys. Chem. 1961, 65 (2), 229-235.

81. Connelly, N. G.; Geiger, W. E., Chemical Redox Agents for Organometallic Chemistry. Chem. Rev. 1996, 96, 877-910.

82. Cardona, C. M.; Li, W.; Kaifer, A. E.; Stockdale, D.; Bazan, G. C., Electrochemical Considerations for Determining Absolute Frontier Orbital Energy Levels of Conjugated Polymers for Solar Cell Applications. Adv. Mater. 2011, 23 (20), 2367-2371.

83. Moral, M.; Muccioli, L.; Son, W. J.; Olivier, Y.; Sancho-García, J. C., Theoretical Rationalization of the Singlet-Triplet Gap in OLEDs Materials: Impact of Charge-Transfer Character. J. Chem. Theory Comput. 2015, 11 (1), 168-177.

84. Adamo, C.; Barone, V., Toward Reliable Density Functional Methods Without Adjustable Parameters: The PBE0 Model. J. Chem. Phys. 1999, 110 (13), 6158-6170.

85. Ditchfield, R.; Hehre, W. J.; Pople, J. A., Self-Consistent Molecular-Orbital Methods. IX. An Extended Gaussian-Type Basis for Molecular-Orbital Studies of Organic Molecules. J. Chem. Phys. 1971, 54 (2), 724-728.

86. Hirata, S.; Head-Gordon, M., Time-Dependent Density Functional Theory Within the TammDancoff Approximation. Chem. Phys. Lett. 1999, 314 (3), 291-299.

87. Geng, Y.; D'Aleo, A.; Inada, K.; Cui, L.-S.; Kim, J. U.; Nakanotani, H.; Adachi, C., Donor-oAcceptor Motifs: Thermally Activated Delayed Fluorescence Emitters with Dual Upconversion. Angew. Chem. Int. Ed. 2017, 56 (52), 16536-16540.

88. Frisch, M. J.; Trucks, G. W.; Schlegel, H. B.; Scuseria, G. E.; Robb, M. A.; Cheeseman, J. R.; Montgomery, J. A.; Vreven, T.; Kudin, K. N.; Burant, J. C.; Millam, J. M.; Iyengar, S. S.; Tomasi, J.; Barone, V.; Mennucci, B.; Cossi, M.; Scalmani, G.; Rega, N.; Petersson, G. A.; Nakatsuji, H.; Hada, M.; Ehara, M.; Toyota, K.; Fukuda, R.; Hasegawa, J.; Ishida, M.; Nakajima, T.; Honda, Y.; Kitao, O.; Nakai, H.; Klene, M.; Li, X.; Knox, J. E.; Hratchian, H. P.; Cross, J. B.; Bakken, V.; Adamo, C.; Jaramillo, J.; Gomperts, R.; Stratmann, R. E.; Yazyev, O.; Austin, A. J.; Cammi, R.; Pomelli, C.; Ochterski, J. W.; Ayala, P. Y.; Morokuma, K.; Voth, G. A.; Salvador, P.; Dannenberg, J. J.; Zakrzewski, V. G.; Dapprich, S.; Daniels, A. D.; Strain, M. C.; Farkas, O.; Malick, D. K.; Rabuck, A. D.; Raghavachari, K.; Foresman, J. B.; Ortiz, J. V.; Cui, Q.; Baboul, A. G.; Clifford, S.; Cioslowski, J.; Stefanov, B. B.; Liu, G.; Liashenko, A.; Piskorz, P.; Komaromi, I.; Martin, R. L.; Fox, D. J.; Keith, T.; Laham, A.; Peng, C. Y.; Nanayakkara, A.; Challacombe, M.; Gill, P. M. W.; Johnson, B.; Chen, W.; Wong, M. W.; Gonzalez, C.; Pople, J. A., Gaussian 03, Revision C.02.

89. Noda, H.; Chen, X.-K.; Nakanotani, H.; Hosokai, T.; Miyajima, M.; Notsuka, N.; Kashima, Y.; Brédas, J.-L.; Adachi, C., Critical Role of Intermediate Electronic States for Spin-Flip Processes in Charge-Transfer-Type Organic Molecules with Multiple Donors and Acceptors. Nat. Mater. 2019, 18 (10), 1084-1090. 
90. Chandross, E. A.; Sonntag, F. I., Chemiluminescent Electron-Transfer Reactions of Radical Anions. J. Am. Chem. Soc. 1966, 88 (6), 1089-1096.

91. Coehoorn, R.; Bobbert, P. A.; van Eersel, H., Förster-Type Triplet-Polaron Quenching in Disordered Organic Semiconductors. Phys. Rev. B 2017, 96 (18).

92. Tokel-Takvoryan, N. E.; Hemingway, R. E.; Bard, A. J., Electrogenerated Chemiluminescence. XIII. Electrochemical and Electrogenerated Chemiluminescence Studies of Ruthenium Chelates. J. Am. Chem. Soc. 1973, 95 (20), 6582-6589.

93. Tokel, N. E.; Bard, A. J., Electrogenerated Chemiluminescence. IX. Electrochemistry and emission From Systems Containing Tris(2,2'-bipyridine)ruthenium(II) Dichloride. J. Am. Chem. Soc. 1972, $94(8), 2862-2863$.

94. Wallace, W. L.; Bard, A. J., Electrogenerated Chemiluminescence. 35. Temperature Dependence of the ECL Efficiency of Ru(bpy $)_{3}{ }^{2+}$ in Acetonitrile and Evidence for Very High Excited State Yields from Electron Transfer Reactions. J. Phys. Chem. 1979, 83 (10), 1350-1357.

95. Lytle, F. E.; Hercules, D. M., Chemiluminescence From The Reduction of Aromatic Amine Cations and Ruthenium(III) Chelates. Photochem. Photobiol. 1970, 13, 123-133.

96. Saiki, H.; Takami, K.; Tominaga, T., Diffusion of Porphyrins and Quinones in Organic Solvents. Phys Chem Chem Phys 1998, 1, 303-306.

97. Barbante, G. J.; Hogan, C. F.; Wilson, D. J.; Lewcenko, N. A.; Pfeffer, F. M.; Barnett, N. W.; Francis, P. S., Simultaneous Control of Spectroscopic and Electrochemical Properties in Functionalised Electrochemiluminescent Tris(2,2'-bipyridine)ruthenium(II) Complexes. Analyst 2011, 136 (7), 1329-38. 98. Parker, C. A.; Hatchard, C. G., Delayed Fluorescence From Solutions of Anthracene and Phenanthrene. P. R. Soc. Lond. A Mat. 1962, 269 (1339), 574-584.

99. Grüne, J.; Bunzmann, N.; Meinecke, M.; Dyakonov, V.; Sperlich, A., Kinetic Modeling of Transient Electroluminescence Reveals TTA as an Efficiency-Limiting Process in Exciplex-Based TADF OLEDs. J. Phys. Chem. C 2020, 124 (47), 25667-25674.

100. Niwa, A.; Haseyama, S.; Kobayashi, T.; Nagase, T.; Goushi, K.; Adachi, C.; Naito, H., TripletTriplet Annihilation in a Thermally Activated Delayed Fluorescence Emitter Lightly Doped in a Host. Appl. Phys. Lett. 2018, 113 (8), 083301-083305.

101. Masui, K.; Nakanotani, H.; Adachi, C., Analysis of Exciton Annihilation in High-Efficiency Sky Blue Organic Light-Emitting Diodes with Thermally Activated Delayed Fluorescence. Org. Electron. 2013, 14 (11), 2721-2726.

102. Feldberg, S. W., A Possible Method for Distinguishing between Triplet-Triplet Annihilation and Direct Singlet Formation in Electrogenerated Chemiluminescence. J. Phys. Chem. 1966, 70 (12), 39283930.

103. Miao, W., Electrogenerated Chemiluminescence and Its Biorelated Applications. Chem. Rev. 2008, 108, 2506-2553.

104. Cormier, M. J.; Hercules, D. M.; Lee, J., Chemiluminescence and Bioluminescence. 1973. 105. Rosenmund, J.; Doblhofer, K., The Effects of Uncompensated Solution Resistance and Rate of the Homogenous Electron Transfer Reaction on Electrochemiluminescence Transients. J. Electroanal. Chem. 1995, 396 (1-2), 77-83.

106. Kabe, R.; Adachi, C., Organic Long Persistent Luminescence. Nature 2017, 550 (7676), 384-387.

107. Tan, S.; Jinnai, K.; Kabe, R.; Adachi, C., Long-Persistent Luminescence From an Exciplex-Based Organic Light-Emitting Diode. Adv. Mater. 2021, e2008844.

108. Jinnai, K.; Nishimura, N.; Adachi, C.; Kabe, R., Thermally Activated Processes in an Organic Long-Persistent Luminescence System. Nanoscale 2021, 13 (18), 8412-8417.

109. Li, W.; Li, Z.; Si, C.; Wong, M. Y.; Jinnai, K.; Gupta, A. K.; Kabe, R.; Adachi, C.; Huang, W.; Zysman-Colman, E.; Samuel, I. D. W., Organic Long-Persistent Luminescence from a Thermally Activated Delayed Fluorescence Compound. Adv. Mater. 2020, 32 (45), e2003911.

110. Cornil, J.; dos Santos, D. A.; Crispin, X.; Silbey, R.; Bredas, J. L., Influence of Interchain Interactions on the Absorption and Luminescence of Conjugated Oligomers and Polymers: A QuantumChemical Characterization. J. Am. Chem. Soc. 1998, 120, 1289-1299. 
111. Tourneur, P.; Lucas, F.; Quinton, C.; Olivier, Y.; Lazzaroni, R.; Viville, P.; Cornil, J.; Poriel, C., White-Light Electroluminescence From a Layer Incorporating a Single Fully-Organic Spiro Compound with Phosphine Oxide Substituents. J. Mater. Chem. C 2020, 8 (41), 14462-14468.

112. Newcomb, R.; Bangsund, J. S.; Hershey, K. W.; Rathwell, D. C. K.; Na, H. Y.; Jeon, J. H.; Trefonas, P.; Holmes, R. J., Role of Host Excimer Formation in the Degradation of Organic LightEmitting Devices. Appl. Phys. Lett. 2020, 116 (6), 063302-0633028.

113. Bui, T. T.; Goubard, F.; Ibrahim-Ouali, M.; Gigmes, D.; Dumur, F., Thermally Activated Delayed Fluorescence Emitters for Deep Blue Organic Light Emitting Diodes: A Review of Recent Advances. Appl. Sci.-Basel 2018, 8 (4), 494-517.

TOC

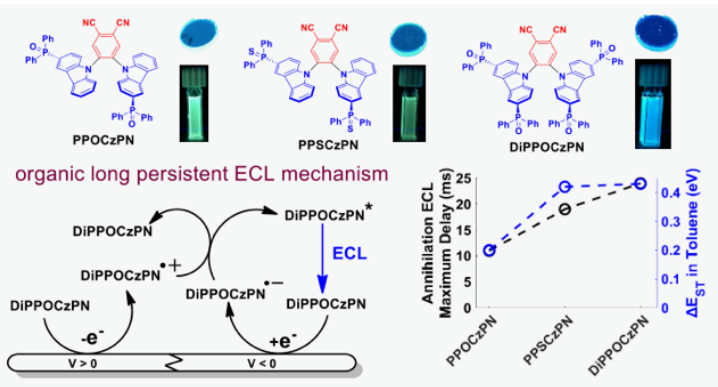

